\title{
La universidad inteligente \\ La transición de los LMS a los Sistemas Inteligentes de Aprendizaje en Educación Superior
}

\author{
The smart university \\ The transition from Learning Management Systems (LMS) to Smart Learning \\ Systems (SLS) in Higher Education
}

\author{
Miguel Zapata-Ros \\ Universidad de Murcia. Murcia, España. \\ mzapata@um.es
}

\begin{abstract}
Resumen
Como sucede en otros ámbitos, también en la Educación Superior se detecta que el uso de la Inteligencia Artificial (IA) puede ser, y en algunos casos está siendo, un potente medio de inclusión a través del apoyo adaptativo en la ayuda pedagógica y en la entrega de recursos. Además puede hacerlo de forma sensible, relevante y pertinente con la situación personal y grupal de aprendizaje de los estudiantes, como respuesta a su demanda de conocimientos y para el desarrollo de sus habilidades.

Se necesita pues un marco de modelo pedagógico y de diseño instruccional, que integre a los alumnos en los nuevos entornos y oriente esa ayuda a unos resultados de aprendizaje comunes a todos y deseables. Planteamos igualmente la necesidad de un análisis de las condiciones necesarias para su validación. Por último, proponemos, a través de análisis basados en experiencias, respuestas concretas ante la insuficiencia de políticas instituciones que contemplen modalidades de integración y sus repercusiones.
\end{abstract}

\section{Palabras clave}

Aprendizaje inteligente, sistemas inteligentes de gestión del aprendizaje, aprendizaje adaptativo, aprendizaje consciente del contexto, educación superior

\begin{abstract}
As it happens in other fields, also in Higher Education it is detected that the use of Artificial Intelligence (AI) can be, and in some cases is being, a powerful medium of inclusion through adaptive support in pedagogical help and in the delivery of resources. And to do it in a sensitive, relevant and pertinent way with the personal and group situation of students' learning, in response to their demand for knowledge and for the development of their skills.

There is a need for a framework of pedagogical model and instructional design that integrates students and guides this help to common and desirable learning outcomes. We also raise the need for an analysis of the conditions necessary for its validation. Finally, we propose, through analysis based on experiences, concrete answers to the insufficiency of institutional policies that contemplate modalities of integration and their repercussions.
\end{abstract}

Keywords.-

Smart learning, Smart learning systems, adaptive learning, higher education, context-aware learning 
"Durante treinta años he asistido a conferencias donde oradores han hablado con diapositivas que comparan las imágenes de un aula de principios del siglo 20 con otra de hoy, y deliberadamente han preguntado: '¿por qué hay tan pocos cambios' La variante moderna de esa pregunta es algo así como esto: las tecnologías inteligentes ya han transformado muchas partes de nuestras vidas - desde la forma en que hoy reservamos un taxi. Podría parecer razonable que la IA también influyera significativamente en lo que enseñamos y en lo que aprendemos, así como la forma en que lo hacemos. Y sin embargo ..."

Sir Michael Barber

\section{Introducción}

Hace falta una respuesta ante un hecho indiscutible: El uso de la IA como un potente medio de adaptabilidad y de inclusión del apoyo de la entrega de ayuda y de recursos de forma relevante y pertinente a la situación personal y grupal de aprendizaje y a la demanda de conocimientos y de habilidades de los estudiantes.

Hay una necesidad de un marco de modelo pedagógico y de diseño instruccional que integre a los alumnos y oriente esa ayuda a unos resultados de aprendizaje comunes y deseables. Planteamos igualmente la necesidad de un análisis de las condiciones necesarias para su validación. Por último, en este trabajo proponemos, a través de análisis basados en experiencias, respuestas concretas ante la insuficiencia de políticas instituciones que contemplen modalidades de integración y sus repercusiones.

A partir de investigaciones sobre el tema, vemos necesarias propuestas de indicadores sobre calidad en entornos sociales de aprendizaje, como resultados procesados y conclusiones obtenidas de investigaciones y experiencias.

Estamos acostumbrados a una literatura que enfatiza las posibilidades de la educación inteligente, y de los big data combinados con los algoritmos de respuesta para crear 
oportunidades únicas y sin precedentes a las organizaciones académicas para un capacitación en términos de estándares más altos y de enfoques innovadores. Sin embargo se carece de propuestas pedagógicas sistematizadas

En definitiva se trata de ver, a este propósito, qué hay en presencia y disponible en cuanto a enfoques teóricos, prácticas y experiencias sobre

(1) estrategias de aprendizaje y enseñanza: pedagogía inteligente,

(2) servicios altamente tecnológicos y singulares basados en entornos de este tipo, tanto para estudiantes locales, en el campus, como para estudiantes remotos, en línea,

(3) configuraciones de aulas inteligentes innovadoras con fácil interacción local/remota de alumno con profesores y centros y para la colaboración local/remota entre alumnos,

(4) diseño y desarrollo de contenidos multimedia-enriquecidos basado en la Web, con presentaciones interactivas, videoconferencias, cuestionarios y pruebas interactivas basadas en la web que admitan evaluación instantánea e inteligente del conocimiento

(5) otras affordances y entornos gestionados con tecnología y software de respuesta inteligente.

Queremos pues plantear una visión sobre la evolución de los LMS hacia los SLE y dar cabida al debate sobre las perspectivas y las tendencias en presencia, con una mirada a algunos resultados de proyectos de investigación y de experiencias en curso. También daremos cabida a trabajos que tengan como objetivos crear una taxonomía de educación universitaria inteligente e identificar sus principales características y componentes.

Los avances en la tecnología en los últimos años han cambiado los comportamientos de aprendizaje de los alumnos y los métodos de enseñanza con nuevas metodologías ambientes y recursos. Esta situación ha dado lugar a nuevos retos: Una mayor presencia y posibilidad del aprendizaje informal, los recursos ubicuos con la posibilidad no sólo de que el alumno acceda desde cualquier sitio a las fuentes y recursos de aprendizaje, y en general de conocimiento, sino también de que ese acceso, el alumno, lo haga ayudado en el aprendizaje por el sistema, con conocimiento de su situación de aprendizaje merced a los ingentes datos que sobre sus hábitos, estilos y formas de aprender se tienen. Estos avances han dado lugar a varios retos que enfrentan los sistemas educativos actuales. Entre ellos se encuentran un más amplio enfoque y consideración del aprendizaje informal, una brecha cada vez mayor de los conocimientos previos de los estudiantes a su acceso a las aulas y una falta de correspondencia entre las opciones de carreras que se ofrecen a los individuos y las que demanda el desarrollo de la fuerza de trabajo.

En este trabajo queremos echar una mirada a esa situación para ver qué nos ofrecen los llamados entornos inteligentes de aprendizaje y la nueva forma de aprender, el aprendizaje inteligente en ese contexto de desafíos y para aprovechar mejor lo que conocemos sobre cómo aprenden los individuos.

No se quiere dar un salto en el vacío. Para ello plantemos un punto de vista realista que permita enlazar los actuales entornos de aprendizaje con lo que los recursos inteligentes nos proporcionan.

Eso posiblemente se logre mediante una re-ingeniería de las estructura tecnológicas y los cambios necesarios de los sistemas universitarios actuales para mejorar la integración de estas nuevas tecnologías con un con el cambio pedagógico adecuado.

La base del desarrollo de los entornos inteligentes es posible merced a dos factores (Hwang, 2014): 
a) El rápido progreso de las tecnologías móviles, de comunicación inalámbrica, y

b) Los procedimientos/affordances/tecnologías de detección que ha permitido el desarrollo de entornos de aprendizaje ubicuo (u-learning) que, sensibles al contexto, son capaces de detectar el estado de aprendizaje en el mundo real de los estudiantes y las características de los contextos ambientales.

En consecuencia, y ateniéndose a los principios del aprendizaje y de intervención educativa en cuanto a pertinencia y relevancia de la ayuda, y en cuanto a la forma de diseñar la entrega de recursos y contenidos, la información apropiada en cada caso se puede proporcionar a estudiantes individuales en el lugar correcto y en el momento adecuado para ello. Recordemos que los procesos instruccionales son procesos eminentemente situacionales (Reigeluth y Carr Chellman, 2009; Reigeluth, 2016).

Sin embargo, los investigadores han indicado que, para ayudar a los estudiantes a aprender en contextos del mundo real de manera inteligente, se deben tener en cuenta más factores al diseñar y desarrollar sistemas de aprendizaje.

En este sentido, en este trabajo se tratará de adecuar la ayuda inteligente, partiendo de lo que es, la definición, y de los criterios de los entornos de aprendizaje inteligentes (con énfasis en la ubicuidad y en la detección) a lo que dicen las teorías y principios clásicos del aprendizaje.

Así trataremos

a) El estado del arte de la cuestión y una sucinta revisión de la literatura

b) Qué es el aprendizaje inteligente. Diferencias entre aprendizaje inteligente, aprendizaje contextualizado y aprendizaje inclusivo.

c) Qué son los entornos inteligentes de aprendizaje. Grandes datos, analíticas y detección, sistemas de recomendación tutorial. Innovaciones pedagógicas en EIA. Innovaciones tecnológicas favorables al aprendizaje inteligente. $Y$ cómo organizar la enseñanza en EIA.

d) Por qué la universidad tiene que ser sensible y adoptar estas innovaciones

Los entornos inteligentes en el contexto de la evolución de la enseñanza abierta universitaria.

Cómo la inteligencia artificial puede beneficiar a las universidades

e) El marco teórico y la pedagogía de un entorno de aprendizaje inteligente.

f) Ejemplos y experiencias

g) Discusión. Cuestiones de investigación del aprendizaje inteligente

h) Conclusiones

\section{El estado del arte de la cuestión y una sucinta revisión de la literatura}

La tecnología ha ofrecido una línea de avance a la atención y a la entrega de contenidos a los alumnos. De manera que como después justificaremos más detenidamente, entenderemos que la línea de progreso en el aprendizaje inteligente y en la creación y uso eficiente de entornos inteligentes de aprendizaje la marca la línea de de adaptabilidad, es decir algo que va 
más allá de la tutoría, es una tutoría contextualizada y después una tutoría inteligente que da respuestas al alumno detectando sus necesidades, en todo lugar y en todo momento, con el auxilio de la tecnología ubicua, con sistemas de detección y de respuesta, con sistemas de recomendación. Por tanto veremos cómo progresa la elaboración de una teoría pedagógica en esta línea

\section{Sistemas adaptativos y tutorías inteligentes}

Desde este punto de vista, de lo que es inteligente, han proliferado en el contexto internacional de una forma ciertamente notable, no así en nuestro país, los trabajos de investigadores tanto en el campo de la computación, de la ingeniería educativa, como en el de la educación, no así las prácticas consolidadas y mucho menos las institucionales, si hacemos excepción de la Universidad de Arizona (Clark, February 19, 2016) y algunas pocas más.

Hacer que los sistemas de aprendizaje tradicionales, apoyados por los LSM, sean inteligentes ha sido pues el objetivo de numerosos investigadores en los campos de la informática y la educación.

Empezaremos por los sistemas inteligentes de tutoría (SIT o en inglés intelligent tutoring systems ITSs o ITS) que incorporan técnicas de inteligencia artificial en aplicaciones educativas. Con estos criterios de evolución de la adaptatividad y de la contextualización, y con este nombre, son tratados por Martens y Uhrmacher (2002) y por van Seters y otros (van Seters et al, 2012).

Basándose en que el objetivo de desarrollar los STI es ayudar a los alumnos a aprender, y que lo hacen con elementos que guían la adaptación de las interfaces de aprendizaje y de los recursos, a los STI también se les llama "sistemas de aprendizaje adaptativo" (Graf et al., 2010; Kinshuk y Lin, 2003), o "adaptive learning systems" (ALS), en función de estas funciones de ayuda contextualizada.

Estos sistemas llevan, con el auge de las redes y de la World Wide Web, que implementan y extienden los mecanismos de ayuda, haciéndolos colaborativos y de mucha más confluencia de recursos, a la implementación y al estudio de sistemas de aprendizaje tutorizado en la web. $Y$ entonces se llaman sistemas de aprendizaje basados en la web (Karampiperis y Sampson, 2005).

\section{Aprendizaje ubicuo y aprendizaje inteligente}

Otro pilar de la asistencia, de respuesta inteligente, es la tecnología ubicua utilizada con este fin. El rápido avance de las redes de comunicación wifi, $4 \mathrm{G}, \ldots$ y el uso de los smartphones han permitido a los alumnos también acceder a recursos digitales e interactuar con sistemas informáticos sin estar limitados ni por ubicación ni por tiempo. Esto ha sido estudiado en esta perspectiva por Hwang y Chang ( 2011) y con criterios de calidad basada en el aprendizaje por Zapata-Ros (2012 y 2015). Los autores Ilaman a este enfoque de aprendizaje, que utiliza tecnologías de comunicación móviles e inalámbricas, "aprendizaje móvil" (Sharples et al. 2009 ]) o "aprendizaje ubicuo" (Zapata-Ros, 2012 y 2015). El otro pilar son las tecnologías de detección: GPS (Sistema de Posicionamiento Global), RFID (Identificación de Radio Frecuencia), QR (Respuesta Rápida),... Ellas ha permitido que "los sistemas de aprendizaje detecten las ubicaciones y contextos del mundo real de los estudiantes" (Hwang, Tsai y Yang, 
2008) y los sistemas de recomendación (Pazzani \& Billsus, 2007; Chen, Cheng \& Chuang, 2008) puedan hacer propuestas basándose en ellas.

Si estas posibilidades son utilizadas para organizar la enseñanza, la ayuda pedagógica y la tutoría se llama "aprendizaje contextualizado" (Hwang, Tsai y Yang, 2008). Es un enfoque del aprendizaje que emplea tecnologías móviles, inalámbricas, de comunicación y detección para permitir a los estudiantes interactuar con el mundo real y los objetos del mundo digital, se consigue un "conocimiento ubicuo consciente del contexto" (Hwang, Tsai y Yang, 2008).

Hay numerosos estudios que han evidenciado los beneficios de estos enfoques y han enfatizado la relevancia de que los estudiantes aprendan del mundo real con acceso a recursos digitales que utilizan en sus actividades y son asistidos por sus profesores en el aprendizaje (Bomsdorf, 2005; Chu et al, 2010). Ha cambiado pues desde el enfoque del aprendizaje ayudado por la tecnología en general y el del aprendizaje basado en la web, hacia el del aprendizaje móvil y sobre todo hacia el del "aprendizaje ubicuo consciente del contexto", como lo indica Liu y Hwang (2010).

\section{Tutoría inteligente y aprendizaje adaptativo}

Sobre el desarrollo de tutorías inteligentes en el contexto de aprendizaje adaptativo para apoyar a los estudiantes en función de su estado de aprendizaje y otros factores personales, como son su forma de progresar en el aprendizaje, los niveles de conocimientos previos, los estilos de aprendizaje, estilos cognitivos y preferencias, han escrito Mampadi, Chen, Ghinea, y Chen (2011); Papanikolaou, Grigoriadou, Magoulas y Kornilakis (2002) y Yang et al. (2013a, b).

Sobre la estructura y los elementos de que consta un sistema clásico de tutoría inteligente, ha escrito Clancey ( 1984).

Sobre sistemas de aprendizaje adaptativo basados en la web, también llamados sistemas hipermedia adaptativos de educación o hipermedia adaptativo, han escrito Kinshuk et al. (2012), y Specht et al. (1997). Y sobre sistemas basados en la web que tienen potencial de proporcionar apoyo de aprendizaje personalizado y orientación a los estudiantes en función de sus características personales o rendimiento de aprendizaje han escrito Chiou et al. (2010) y Hwang (1998). Sobre personalización con rutas de aprendizaje mediante la adaptación del contenido de aprendizaje con la estructura de enlace han escrito Tseng y otros (2008a, b), Brusilovsky (2001), Kinshuk y otros (2012) y Yang y otros (2013a, b).

Y por último, han investigado sobre sistemas adaptativos e inteligentes basados en la web y difundido los resultados de sus trabajos Tseng et al. (2008a, b), González e Ingraham (1994), Papanikolaou et al. (2002), Karampiperis y Sampson (2005) y Martens (2006).

\section{Sobre el "aprendizaje ubicuo sensible al contexto"}

Han justificado este planteamiento y esta perspectiva, la importancia y la necesidad de situar a los estudiantes en escenarios de aprendizaje del mundo real, como una variante específica del conocido y estudiado como aprendizaje situado (Merrill, 1991) (Reigeluth, 2016), y lo han 
identificado como tal Lave (1991), Hung et al. (2013 y 2014) y Brown et al. (1989).

Entre los investigadores que han intentado desarrollar entornos de aprendizaje que combinan contextos del mundo real y recursos del mundo digital para proporcionar a los estudiantes experiencias directas del mundo real, con apoyo en las teorías del aprendizaje podemos citar a (Minami et al. (2004), Hung et al. (2014), Wu y otros (2013a, b).

Sobre el aprendizaje ubicuo consciente del contexto y sobre su naturaleza como enfoque que permite a los estudiantes aprender del mundo real con el apoyo de tecnologías móviles, de comunicación inalámbrica, y de tecnologías de detección han escrito Hwang et al. (2008), Tsai et al. (2012) y además con la concurrencia de herramientas de recomendación podemos citar a Chen, Cheng \& Chuang (2008).

Sobre apoyo pedagógico en función de sistemas de detección en un entorno de aprendizaje ubicuo consciente del contexto, han investigado y han escrito Chu et al. (2010), Joiner et al. (2006), Rogers et al. (2005).

En este capítulo se pueden citar ejemplos concretos:

Ogata y Yano (2004) desarrollaron un sistema de aprendizaje ubicuo consciente del contexto con GPS para la enseñanza y la práctica de japonés

Hwang y otros (2012) desarrollaron un sistema de aprendizaje con RFID para enseñar a utilizar instrumentos científicos, en un parque científico, a través de la asignación de varias tareas "operativas" y de la evaluación de sus resultados operativos.

Sobre la importancia de proporcionar estrategias de aprendizaje efectivas, herramientas y apoyo de aprendizaje personalizado en sistemas de aprendizaje ubicuo conscientes del contexto, es decir, sobre incorporar tutoría inteligente o técnicas de aprendizaje adaptativo, han escrito Hung et al. (2014), Wu et al. (2013a, b).

Citamos, aunque después se ha cuestionado todo, lo concerniente a los estilos de aprendizaje como criterio orientador del diseño instruccional y de la organización de las actividades y procesos educativos, el trabajo de Hsieh et al. (2011) que presentaron un sistema de aprendizaje adaptativo para guiar a los estudiantes a realizar observaciones en un jardín de mariposas en función de sus estilos de aprendizaje. Sí tiene interés como ejemplo de tutoría inteligente o de técnicas de aprendizaje adaptativo a escenarios de aprendizaje del mundo real.

De forma general todo lo tratado en esta recensión está desarrollado y muy bien organizado, con detalles, en los trabajos de Hwang (2014) y de Chen, Cheng y Chew(2016).

Hasta ahora hemos hecho una revisión de la literatura basada en conceptos, a partir de ahora se revisan con relación a temas y a prácticas, es decir se revisan varios temas de investigación relacionados con el desarrollo de sistemas de aprendizaje inteligente que pueden ayudar a los estudiantes a aprender de forma eficiente y efectiva.

Ya nos hemos acercado al "aprendizaje ubicuo consciente del contexto". Los sistemas inteligentes de tutoría y adaptación y los sistemas de aprendizaje ubicuo conscientes del contexto son sistemas que pretenden proporcionar un soporte de aprendizaje personalizado 
basado en las preferencias de los estudiantes, el estado de aprendizaje, los factores personales y las características de los contenidos de aprendizaje y entornos de aprendizaje.

\section{Mindtools}

Los Mindtools son affordances que implican a los estudiantes en actividades cognitivas de orden superior y en el pensamiento significativo durante el proceso de aprendizaje según Jonassen, Carr y Yueh (1998). Sirven para ayudar a los estudiantes a comprender y organizar el conocimiento, para resolver problemas y para hacer inferencias basadas en lo que han aprendido. En los entornos conscientes de contexto es importante suministrar estas herramientas de forma oportuna y relevante para que se puedan abordar diferentes tareas de aprendizaje o resolver diferentes tipos de problemas en el momento adecuado y en el correcto contexto. Sobre estas herramientas en este contexto han escrito Chu, Hwang y Tsai (2010) y Hwang, Hung, Chen y Liu (2014).

\section{Aprendizaje inteligente}

Aprendizaje inteligente es aquél aprendizaje que pueden desencadenar y producir los sistemas de aprendizaje inteligente, y que se puede entender de forma diferenciada por las funciones $y$ efectos que no se pueden realizar en ambientes de aprendizaje adaptativo y de aprendizaje contextualizado, sin excluirlas. El aprendizaje social y el aprendizaje colaborativo de esta forma se puede entender como una subcategoría del aprendizaje contextualizado.

Resumiendo se puede decir que el aprendizaje inteligente es el aprendizaje adaptativo y contextualizado con mecanismos de detección, de respuesta y de recomendación.

Así, como veremos en el apartado siguiente, los "entornos de aprendizaje inteligentes" pueden considerarse, a partir de la definición de Hwang et al (2008), como entornos de aprendizaje apoyados por la tecnología que realizan adaptaciones y brindan el soporte adecuado con la tecnología de detección y de recomendación (por ejemplo, orientación, retroalimentación, consejos o herramientas) en los lugares adecuados y en el momento oportuno, según las necesidades individuales de cada alumno, determinados mediante el análisis de sus comportamientos de aprendizaje, su trayectoria de rendimiento y los contextos en línea y del mundo real en los que se encuentran tanto grupales y sociales como compuesto por los recursos de que se provee o a los que accede, descarga, lee o elabora.

\section{Diferencias entre aprendizaje en entornos inteligentes, aprendizaje por contexto y aprendizaje inclusivo}


Hemos dicho que el aprendizaje inteligente como apoyo a los alumnos en la tutoría y en la entrega de materiales se considera una evolución del aprendizaje adaptativo y del aprendizaje contextualizado, o sensible al contexto.

Por tanto ninguno de los dos puede considerarse como estado óptimo de aprendizaje apoyado por la tecnología, al que llamaremos aprendizaje inteligente en lo sucesivo.

Como veremos igualmente en el apartado siguiente, y consecuentemente con la distinción anterior, un entorno de aprendizaje inteligente no sólo es el que permite a los estudiantes acceder a recursos digitales e interactuar mediante un sistema adecuado de aprendizaje en cualquier lugar $\mathrm{y}$ en cualquier momento, sino que también proporciona activamente $\mathrm{y}$ dinámicamente una guía de aprendizaje, orientaciones, affordances de apoyo o propuestas de actividades de aprendizaje necesarias y adecuadas a los objetivos y a su situación de aprendizajes, en el lugar adecuado, en el momento oportuno y en la forma correcta.

Hay varias características que hacen que una noción tan innovadora de aprendizaje, como es la de aprendizaje inteligente, vaya más allá de la combinación de las dos de donde procede. En lo que sigue se proporcionan elementos de discusión en profundidad para definir claramente el "entorno de aprendizaje inteligente" y su marco teniendo como referencia lo que hace o cómo se produce en relación a sus predecesores, es decir haciendo una comparación entre entornos de aprendizaje adaptativos, entornos de aprendizaje ubicuos conscientes del contexto y sistemas de tutoría inteligentes.

Sobre la base de esos criterios y en ese marco, en la Tabla 1, obtenida a partir del trabajo anterior de Hwang (2014) se presenta una comparación de un entorno de aprendizaje inteligente, un entorno de aprendizaje basado en el contexto y un entorno de aprendizaje adaptativo convencional. Se puede ver que un entorno de aprendizaje inteligente es más que una combinación de u-learning consciente del contexto y aprendizaje adaptativo. En particular, guía a los estudiantes a hacer lo correcto (es decir, las tareas que son más factibles e importantes para el alumno en este momento) de la manera correcta (es decir, con herramientas o estrategias de aprendizaje efectivas) en el momento adecuado y en el lugar apropiado, basándose para ello en el estado de aprendizaje en línea y del mundo real de los estudiantes individuales, así como en sus factores personales.

Tabla 1.- Comparación del aprendizaje inteligente con los sistemas de aprendizaje por contexto y con el aprendizaje adaptativo

\section{Aprendizaje Aprendizaje inteligente contextualizado}

Detecta y tiene en cuenta los contextos del mundo real del alumno

Sí

Sí
Sí

Sí 


\begin{tabular}{|c|c|c|c|}
\hline & $\begin{array}{l}\text { Aprendizaje } \\
\text { inteligente }\end{array}$ & $\begin{array}{l}\text { Aprendizaje } \\
\text { contextualizado }\end{array}$ & $\begin{array}{l}\text { Aprendizaje } \\
\text { adaptativo }\end{array}$ \\
\hline \multicolumn{4}{|l|}{$\begin{array}{l}\text { pueda aplicar lo aprendido con autonomía y } \\
\text { transfiriéndolo a situaciones diferentes }{ }^{1}\end{array}$} \\
\hline $\begin{array}{l}\text { Adapta contenidos y situaciones de aprendizaje para } \\
\text { alumnos individuales }\end{array}$ & Sí & No & Sí \\
\hline $\begin{array}{l}\text { Adapta la interfaz y las affordances de aprendizaje } \\
\text { para alumnos individuales }\end{array}$ & Sí & No & Sí \\
\hline $\begin{array}{l}\text { Adapta tareas u objetivos de aprendizaje para alumnos } \\
\text { individuales, y los criterios de evaluación, cómo } \\
\text { alcanza los logros }\end{array}$ & Sí & No & No \\
\hline $\begin{array}{l}\text { Proporciona comentarios personalizados y } \\
\text { orientaciones }\end{array}$ & Sí & Sí & Sí \\
\hline $\begin{array}{l}\text { Proporciona comentarios personalizados y } \\
\text { orientaciones como resultado de sistemas de } \\
\text { recomendación }\end{array}$ & Sí & No & No \\
\hline $\begin{array}{l}\text { Proporciona orientación de aprendizaje, apoyo y } \\
\text { regulación en la entrega de materiales en todas las } \\
\text { disciplinas }\end{array}$ & Sí & No & No \\
\hline $\begin{array}{l}\text { Proporciona orientación de aprendizaje o apoyo en } \\
\text { todos los contextos (por ejemplo, en las aulas, en los } \\
\text { campus, en la biblioteca, en casa y en la calle) }\end{array}$ & Sí & Sí & No \\
\hline $\begin{array}{l}\text { Recomienda herramientas o estrategias de aprendizaje } \\
\text { específicas para cada situación }\end{array}$ & Sí & No & No \\
\hline $\begin{array}{l}\text { Tiene en cuenta la situación y las condiciones de } \\
\text { aprendizaje en línea de los estudiantes }\end{array}$ & Sí & No & Sí \\
\hline $\begin{array}{l}\text { Tiene en cuenta la situación de aprendizaje en el } \\
\text { mundo real de los estudiantes }\end{array}$ & Sí & Sí & No \\
\hline Facilita tanto el aprendizaje formal como el informal & Sí & Sí & No \\
\hline
\end{tabular}

${ }^{1}$ Según lo que establecen los desarrollos clásicos sobre condiciones de aprendizaje, como son por ejemplo "Las condiciones del aprendizaje" y los "Principios del diseño instruccional" de Gagné $(1965,1985)$ 


\begin{tabular}{|l|l|l|l|} 
& $\begin{array}{l}\text { Aprendizaje } \\
\text { inteligente }\end{array}$ & $\begin{array}{l}\text { Aprendizaje } \\
\text { contextualizado }\end{array}$ & $\begin{array}{l}\text { Aprendizaje } \\
\text { adaptativo }\end{array}$ \\
\hline $\begin{array}{l}\text { Tiene en cuenta los múltiples y diversos factores } \\
\text { personales y ambientales del alumno (en relación con } \\
\text { aspectos relacionados con el aprendizaje. Por ejemplo, } \\
\text { necesidades de aprendizaje, preferencias, hábitos, } \\
\text { horarios y contexto del mundo real) }\end{array}$ & Sí & No & No \\
\hline $\begin{array}{l}\text { Utiliza sistemas de recomendación a partir de lo } \\
\text { detectado, y que se describe en los apartados } \\
\text { anteriores }\end{array}$ & Sí & No & No \\
\hline $\begin{array}{l}\text { Interactúa con los usuarios a través de múltiples } \\
\text { canales (por ejemplo teléfonos inteligentes, } \\
\text { affordances sociales u otros medios informáticos } \\
\text { ubicuos) }\end{array}$ & Sí & Sí & \\
\hline $\begin{array}{l}\text { Brinda apoyo a los alumnos con "adaptación } \\
\text { anticipada" al desarrollo o la ejecución de actividades } \\
\text { en contextos reales y virtuales }\end{array}$ & Sí & Sí & No \\
\hline $\begin{array}{l}\text { Brinda apoyo a los alumnos con "adaptaciones en } \\
\text { progreso" en contextos reales y virtuales }\end{array}$ & No & No \\
\hline
\end{tabular}

\section{Los entornos inteligentes de aprendizaje}

Qué son los entornos inteligentes de aprendizaje. Definición, criterios y marco de un entorno de aprendizaje inteligente.

Así, a partir de lo dicho para los entornos inteligentes de gestión del aprendizaje, y desde la posibilidad de que el aprendizaje sea ubicuo y sensible al contexto, es decir de que pueda crear y detectar y procesar las interacciones entre los estudiantes y de ellos con el ambiente, proporcionando recomendaciones podemos hablar de entornos inteligentes de aprendizaje.

Por tanto, siguiendo lo dicho por Hwang et al (2008) y añadiendo la consideración de las posibilidades de recomendación que permite la tecnología inteligente, podemos decir que los 
"entornos de aprendizaje inteligentes" pueden considerarse como aquellos entornos de aprendizaje apoyados por la tecnología que realizan recomendaciones, adaptaciones de la ayuda al individuo (por ejemplo, orientación, retroalimentación, consejos o affordances), y que proporcionan el soporte adecuado para ello en el lugar en que se produce la necesidad de la ayuda, en el momento oportuno según las necesidades individuales de los alumnos, y en la forma más eficiente. Coordenadas que podrían determinarse mediante el análisis de los comportamientos de aprendizaje, el rendimiento anterior y en proceso y los contextos del estudiante, tanto en línea como del mundo real.

Obviamente una consideración nueva que aparece es la de la distribución del tiempo del alumno, la diferenciación entre ocio y trabajo. En un entorno de aprendizaje no existe una cantidad de tiempo fijo y libre. No porque se le reduzca el tiempo de ocio, que ahora lo tendrá igualmente y posiblemente más, sino que recibirá consejos y aportaciones incluso en su tiempo libre, es como un sabio amigo. Este sistema tiene como objetivo ayudar a los alumnos a aprender incluso cuando tienen otras relaciones o realizan otras actividades. Es el amigo aventajado, o el profesor amigo, que busca oportunidades para asesorar al estudiante en su vida diaria teniendo en cuenta sus necesidades y preferencias. Obviamente esto es una ventaja pero igualmente puede ser considerado como algo intrusivo, en cualquier caso los conceptos de disposición y de distribución del tiempo cambian, como cambian en otros órdenes estos conceptos con relación al trabajo en la sociedad del conocimiento. Y esto tendrá repercusiones evidentes para la configuración de espacios universitarios, espacios de aprendizaje y espacios vitales.

Por tanto podemos visto lo anterior podemos definir un entorno inteligente de aprendizaje como aquél que cumple los criterios potenciales siguientes:

1. Es sensible y consciente del contexto y puede elaborar recomendaciones en función de él. Es decir detecta la situación del alumno en su contexto en el mundo real, y puede proporcionar ayuda al alumno en función de ella.

2. Es adaptativo. Puede ofrecer apoyo instantáneo y adaptado a los alumnos mediante el análisis inmediato de las necesidades, en términos de organizar la instrucción en el nivel más pequeño e inmediato, y hacerlo desde diferentes perspectivas (por ejemplo desde la perspectiva del rendimiento del aprendizaje, conociendo las estrategias de aprendizaje del alumno, su perfil para el trabajo y el estudio, otros factores personales), así como su contexto en línea y el del mundo real (familia, amigos, hábitos,...). Este carácter supone además que puede proporcionar ayuda pedagógica de diverso tipo y activamente, incluidas guías específicas de aprendizaje, comentarios, sugerencias y affordances educativas en función de sus necesidades.

3. Puede modificar, en la misma ejecución del proceso, la interfaz de usuario (es decir, las formas de presentación de la información) y los contenidos de la asignatura. Adaptándolos a los factores personales (por ejemplo, a la forma de leer y reconocer la información que es más eficiente para él y su forma de aprender y a sus preferencias para leer e interpretar información) y al estado de aprendizaje (por ejemplo, 
presentando la información en la forma y en el momento en que es mayor el rendimiento de aprendizaje) de los alumnos individuales. La interfaz de usuario no es necesariamente una computadora convencional, puede ser un teléfono móvil, o un tablet. Los alumnos pueden interactuar con el sistema de aprendizaje a través de dispositivos actuales, por ejemplo, teléfonos inteligentes o tablets, dispositivos que vayan apareciendo, por ejemplo visores de realidad virtual, relojes digitales o incluso, en un futuro no muy lejano sistemas informáticos integrados en objetos cotidianos (Internet of Things, loT). Es un reto pues para la investigación adaptar la interfaz de usuario para satisfacer las necesidades educativas en un entorno de aprendizaje inteligente.

Es la idea de quien esto escribe que los requerimientos pedagógicos de los sistemas se pueden establecer desde un conocimiento sucinto de las funcionalidades de los sistemas inteligentes en otros entornos de lo que los algoritmos de recomendación son capaces de hacer en otros ámbitos $Y$ que su papel culmina ahí. Cualquier otra pretensión quedaría necesariamente inadecuada al poco de producirse y además se produciría de forma pretenciosa al conocer del conocimiento suficiente $y$ en profundidad para saber cuáles son las posibilidades ce los sistemas computacionales.

Por tanto no recomendamos ninguna configuración de sistemas en particular. Sin embargo, dentro del objetivo de poner a disposición de los investigadores lo que hay en la literatura sobre el tema, no podemos evitar incluir la propuesta de Hwang, Chin-Chung y Yang (2008) para que se cumplan estas funciones. Según estos autores el marco de un entorno de aprendizaje inteligente debe contemplar al menos los siguientes módulos:

1. Un módulo de detección de estado de aprendizaje, que detecte el estado del mundo real de los estudiantes (por ejemplo, ubicaciones y comportamientos, hábitos de aprendizaje) y contextos ambientales (por ejemplo horarios, temperatura y humedad) mediante la conexión a algunos dispositivos de detección y de recomendación, y con algoritmos que busquen regularidades.

2. Un módulo de evaluación formativa del rendimiento de aprendizaje que registra todo lo que está sucediendo y los resultados de aprendizaje, valorando cómo inciden los cambios de organización y de carácter instruccional en el total del curso. Por ejemplo análisis y atención del abandono, accesos a los elementos de entrega de contenidos de aprendizaje y resultados, efecto de las medidas que se toman, por ejemplo en la evaluación en el rendimiento global del curso o de los grupos de alumnos etc.

3. Un módulo de tareas de aprendizaje adaptativo. Este módulo, ayudado por una base de datos, asigna tareas de aprendizaje a cada alumno en función de su progreso de aprendizaje (recomendamos en este sentido la metodología docente y de evaluación Mastery Learning, que hemos tratado en otros trabajos (Zapata-Ros, 2015), Brandman (2013) y que se debe originalmente a Bloom (1984)), pero también a otros datos que hay en el sistema sobre rendimiento de aprendizaje, expectativas, intereses, percepciones, aspectos léxicos y, sobre todo, a sus objetivos de aprendizaje en todas las disciplinas. Sobre el carácter adaptativo de las tareas en línea o en el mundo real hay bastante escrito (Hwang et al, 2014). Con la ayuda de este módulo se consigue que los alumnos además trabajen de forma adecuada y adaptada al 
medio donde están, no es igual el repaso de un PDF cuando viaja en el transporte colectivo, que una labor que necesita un ambiente cómodo para consultar escribir, o una actividad de laboratorio o de campo. Los alumnos de esta forma siempre tendrán la oportunidad de hacer las tareas de aprendizaje más factibles e importantes en relación con el contexto, al considerar su estado de aprendizaje y sus factores personales cuando se le recomiendan en el lugar correcto y en el momento adecuado.

4. Un módulo de contenidos de aprendizaje adaptativos. Este módulo proporciona materiales de aprendizaje para los estudiantes basándose para ello en el progreso del aprendizaje y el rendimiento del aprendizaje efectivo (aquí otra vez recomendamos tener en cuenta la metodología de Mastery Learning (Zapata-Ros, 2015; Bloom, 1984)) y en los factores personales de aprendizaje del alumno. Para esto, en la línea de utilizar los resultados de la investigación y de las teorías del aprendizaje recomendamos lo que hay estudiado y elaborado sobre técnicas y teoría de la secuenciación de contenidos, en el contexto de Diseño Instruccional. Todo para recomendar contenidos en función de las actividades que el alumno necesita realizar para conseguir un resultado específico de aprendizaje. Es fundamental pues adaptar las técnicas y algoritmos de recomendación a este tema. El sistema de aprendizaje debe recomendar y organizar materiales de aprendizaje, que propone a través de la interfaz del usuario al alumno, para satisfacer la demanda individual de contenidos de cara a un objetivo específico.

5. El módulo de apoyo de aprendizaje personal es el núcleo del sistema En él deben estar centradas las funciones de asistencia y recomendación al alumno en función de sus necesidades de aprendizaje que detectan el resto de herramientas de detección y procesando las informaciones que sobre él y sobre su contexto disponen las bases de datos del sistema. Como ejemplos, el apoyo al aprendizaje puede consistir en una guía de la tareas de aprendizaje o con documentos, textos, PDF,... con contenidos, una ayuda particular para una tarea, feedback sobre lo que está haciendo la herramienta adecuada para ayudar a su trabajo, retroalimentación sobre su trabajo, o una Mindtools para ayudar a organizar sus ideas.

6. El módulo anterior puede contener otro o estar diferenciado del que gestiona las bases de datos para organizar la ayuda al estudiante. Sería en todo caso el módulo soporte de la ayuda al estudiante. Y lo realmente importante sería el conjunto de bases de datos para mantener y gestionar los perfiles del alumno, los porfolios (carteras de aprendizaje), las guías de aprendizaje (es decir, las fichas que presentan las tareas de aprendizaje para cada unidad temática o para cada tema de aprendizaje), y en general los materiales de aprendizaje, los ejercicios a proponer y las evidencias de logros (elementos que sirven para probar los aprendizajes conseguidos).

A través del análisis de los registros en los porfolios, el sistema tiene constancia y permite analizar los conceptos y las habilidades individuales que los estudiantes han desarrollado y cuáles han de ser mejoradas.

7. Un módulo de evaluación del rendimiento de aprendizaje. Este módulo debe estar basado en los criterios del diseño instruccional y debe relacionar el rendimiento en el aprendizaje con las otras componentes del diseño instruccional: Resultados esperados y deseados de aprendizaje, actividades, tareas, competencias que se desea que el alumno desarrolle, grado 
de logro y de desempeño progresivos, en conexión con el módulo de evaluación adaptativa,... Este módulo evalúa y registra el desempeño de los estudiantes a través de pruebas en línea o en el mundo real.

8. Y por último, un módulo constituido por un motor de inferencia y por una base de conocimientos en el sentido clásico de la programación declarativa (Zapata-Ros, 1990). Mediante el motor de inferencia se determinaría (Hwang, 2014) el "valor" de las tareas, estrategias y herramientas de aprendizaje del candidato, así como sus posibles combinaciones. Y la base de conocimientos estaría constituida por la recopilación del conocimiento y de experiencias de tutoría de proveída por los docentes, tutores y evaluadores. El motor de inferencia también podría contener las reglas para toma de decisiones generadas por el análisis de casos anteriores. El motor de inferencia es un sistema de software de bajo nivel en los sistemas declarativos que toma decisiones mediante reglas (Zapata-Ros, 1990), obtenidas en este caso de las experiencia, aplicadas al caso actual (es decir, el estado del estudiante y los contextos ambientales) en base a las reglas en la base de conocimiento (Wu et al, 2013a, b). 


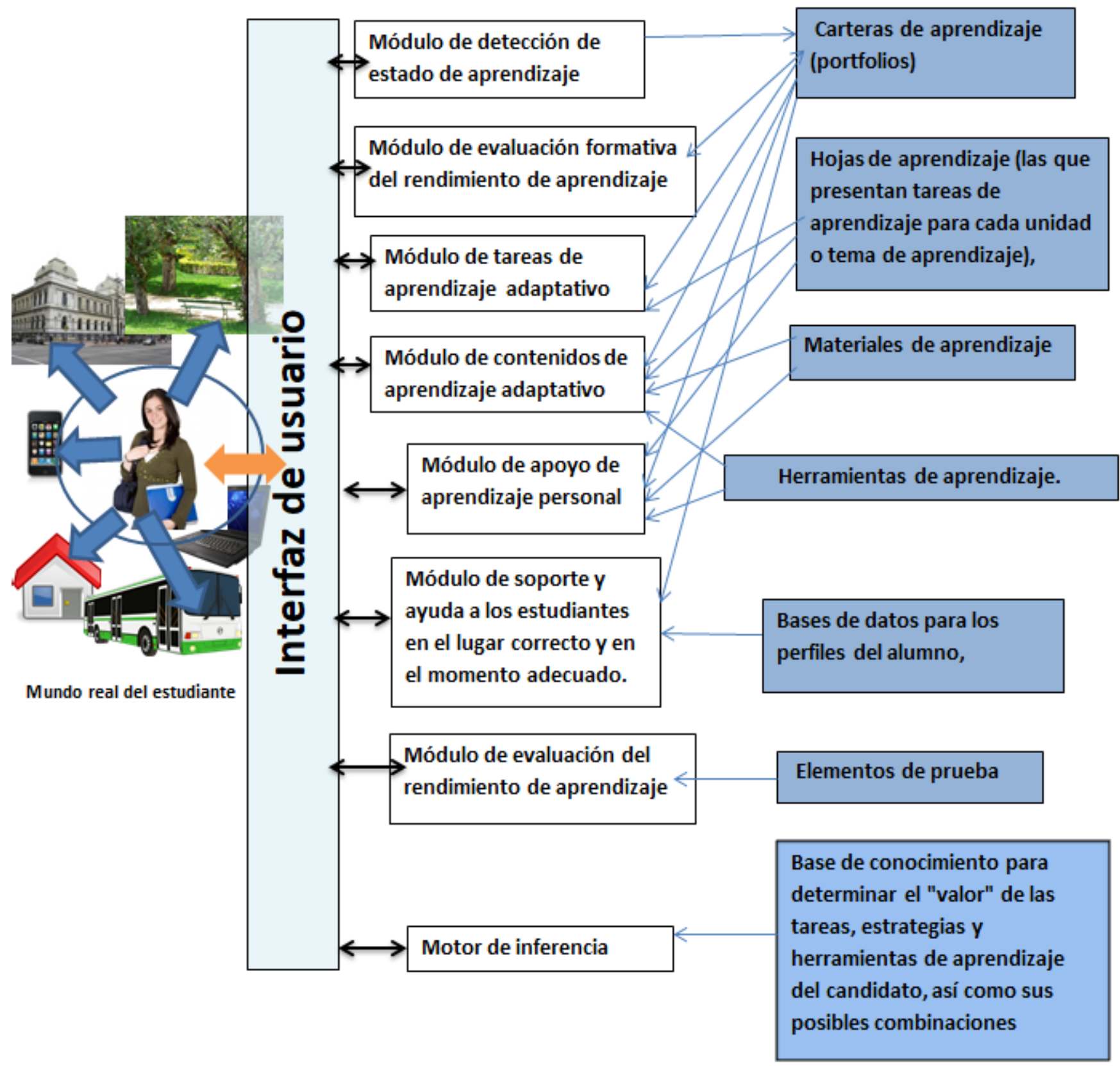

Fig. 1

\section{Por qué la universidad necesita la IA}

\subsection{La evolución de la enseñanza abierta universitaria}

No se puede excluir la universidad inteligente, la que incorpora los sistemas inteligentes de gestión del aprendizaje, de la evolución de la universidad abierta, desde la lógica de su propia concepción original.

En 1997 ya el Further Education Funding Council (FEFC, 1997 p.25; Zapata-Ros, , 2001 p.1) de Gran Bretaña define la educación a distancia como: 
"those on which students study with specially prepared learning materials for their private study and are provided with a marking and comment service for their written work which may be accompanied by some counselling or tutorial support This definition is intended to cover situations where study is essentially home based and there is only occasional contact with the institution"

La educación abierta universitaria tiene tradicionalmente como fin el que se superen las dificultades de condiciones y organización para el aprendizaje (Zapata-Ros, 2001 p.1). Y esto se ha constituido en un objetivo para los sistemas educativos universitarios en las sociedades desarrolladas. Las instituciones, las experiencias que se desarrollan en ellas y centros de investigación se esmeran en indagar qué modalidades de organización, con los usos más adecuados de aplicaciones ---affordances--- son más eficaces para obtener los resultados de aprendizaje propuestos para cada modalidad de estudio, o de rendimiento profesional. Se trata de ofrecer a los profesores y gestores docentes de la enseñanza superior modalidades de diseño instruccional que consigan estos objetivos, que faciliten en definitiva la itinerancia de las situaciones actuales a una enseñanza universitaria abierta.

La evolución de la enseñanza abierta universitaria ha sido objeto de estudio en varios trabajos anteriores. En un artículo (Zapata-Ros, 2017; Zapata-Ros, 2017, December) y en varios posts (Zapata-Ros, 2017)

Tras lo aparecido en ese artículo, los herederos de los MOOC finalmente han evolucionado hacia un nuevo modelo de enseñanza formal universitaria. De forma que tras pasar por varias fases, han llegado a una situación completamente distinta a la inicial: Se han convertido en formas de enseñanza universitaria que otorga títulos oficiales de grado y postgrado, como sucede en los tradicionales. En nada se diferencian de ellos, si pensamos en todos los efectos que aquellos conllevaban.

Lo han hecho la Universidad de Londres y el Imperial College de Londres (a través de Coursera, con su plataforma y su metodología, y The Open University a través de Future Learn. En el primer caso son grados formales y en el segundo son postgrados (másteres) también formales. La universidad disruptiva es un hecho. Cualquier alumno en Alcalá o en Murcia, o en Buenos Aires, o México capital puede obtener un grado (licenciatura) en el Imperial College de Londres como si fuera un MOOC.

Este post está escrito a partir de la noticia de Times Higher Education el 07/03/2018 (Bothwell, March 6, 2018) titulado Coursera ofrecerá títulos de universidades del Reino Unido.

En él se informa (Bothwell, March 6, 2018) de nuevos cursos de la Universidad de Londres y del Imperial College de Londres que incluyen el primer programa de licenciatura de la plataforma y de másteres oficiales también con el soporte y la metodología de Coursera

En particular la plataforma de aprendizaje en línea Coursera se nos dice está lista para ofrecer sus primeros títulos de universidades del Reino Unido, incluido su primer programa de licenciatura: Un grado en Ciencias de la Computación de la Universidad de Londres (Goldsmiths) y un nuevo máster de salud pública en el Imperial College de Londres. 
Además de cuatro nuevos títulos de máster de instituciones estadounidenses, incluidos programas de informática de la Universidad Estatal de Arizona y la Universidad de Illinois en Urbana-Champaign , y títulos de ciencias de la información y salud pública de la Universidad de Michigan. Coursera igualmente anuncia que el número de grados disponibles a través de su plataforma se elevará a diez, a lo largo de este año y en 2019.

Con respecto a esta plataforma, esto significa la culminación de un proceso que se inició con los MOOC, continuó con los MOOC con diseño instruccional de Fink (2003) y clásico, y por último con Credentials, College Credits y Microdegrees. Reflejan pues la evolución de las plataformas de aprendizaje en línea desde sus raíces, ofreciendo cursos gratuitos masivos abiertos en línea, hasta ahora que ofrecen programas acreditados y pagados de títulos oficiales universitarios completos.

Este punto, en el que Coursera lanza un grado, significa un hito, marca la culminación de una fase clave en un proceso. Después vendrán otros grados que ya se han iniciado. Pero antes ya existían títulos oficiales universitarios sobre marcos y con metodologías de plataformas de estudios abiertos online. Antes que los grados del Reino Unido puestos en marcha por Coursera, la gran plataforma con sede en el Reino Unido Future Learn, propiedad de The Open University, lanzó una serie de postgrados en su plataforma y con su estilo, en la Universidad de Coventry el año pasado. Future Learn también ofrece títulos oficiales de másteres en las universidades Deakin y Murdoch en Australia.

Así el esquema de la evolución de la enseñanza abierta universitaria, ahora quedaría pues completo, en este ciclo, con la inclusión de estudios formales de grado y postgrado, en esta línea del tiempo: 


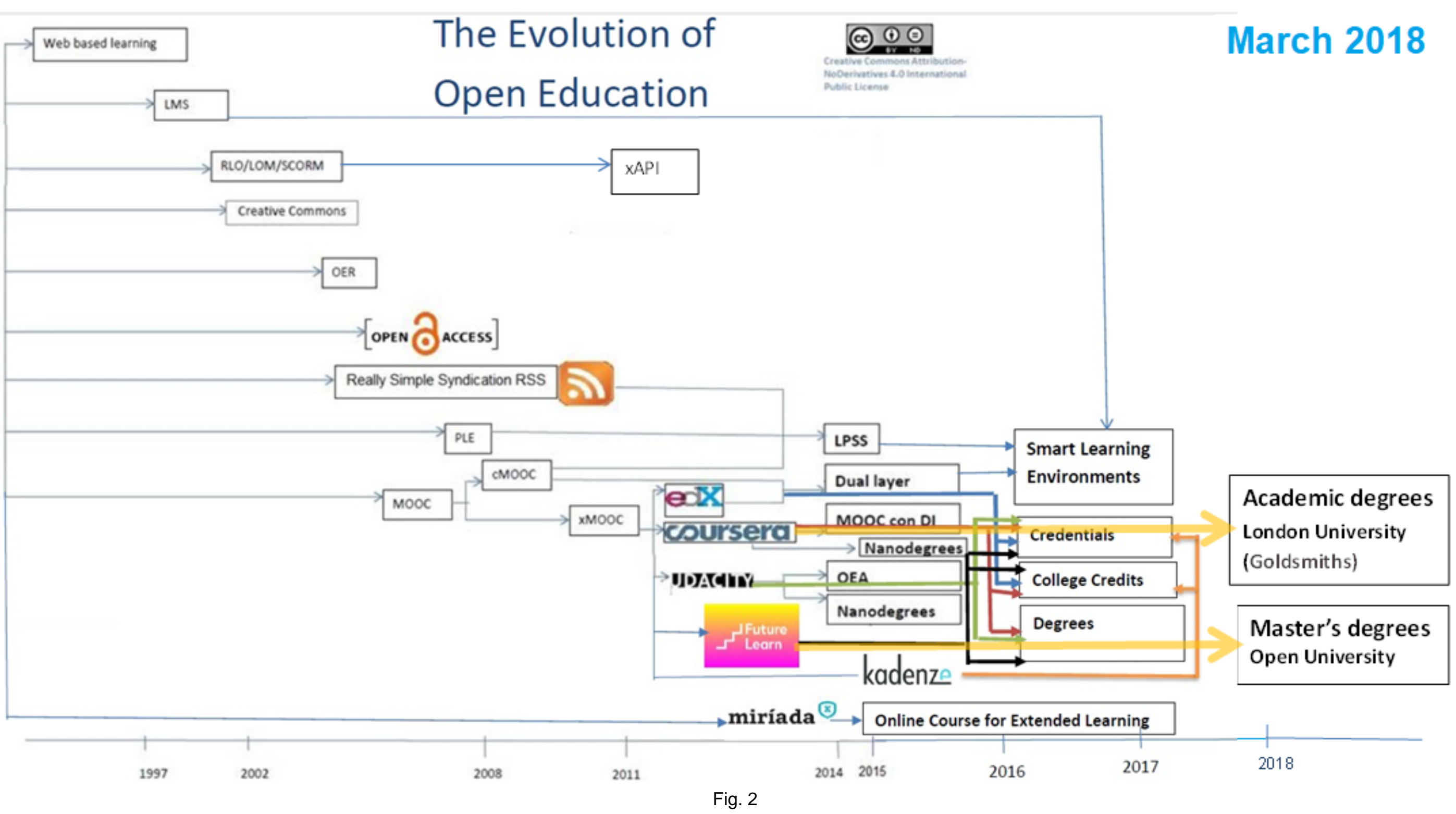


Con lo visto en el apartado anterior el esquema de la evolución de los entornos inteligentes de aprendizaje quedaría:

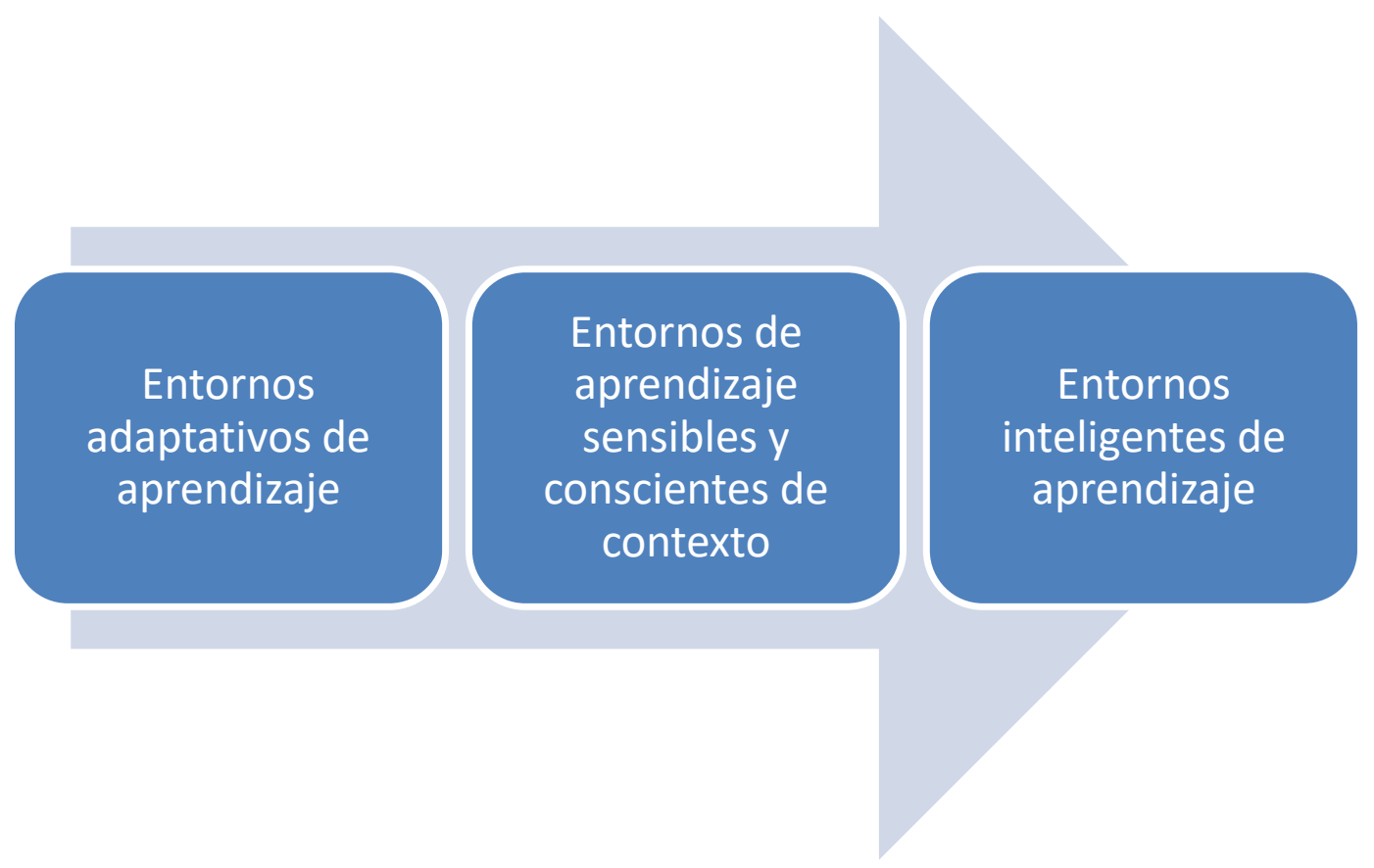

Fig. 3

\subsection{Cómo la inteligencia artificial puede beneficiar a las universidades}

La IA en general, y los sistemas inteligentes de gestión del aprendizaje, como caso particular de los sistemas de aprendizaje adaptativo, en particular, se espera que tengan un enorme efecto a largo plazo en tres campos de la educación universitaria: En la docencia, en el rendimiento de los alumnos y en el abandono escolar.

Como veremos en el apartado de experiencias, estas expectativas han sido confirmadas por los resultados de los cursos realizados en la Universidad Estatal de Arizona en otoño de 2015, y por el correspondiente informe (Clark, February 19, 2016) donde el curso Biology 100 (BIO100), realizado como aprendizaje mixto en la plataforma CogBooks, se estudió en detalle.

Las evidencias obtenidas fueron sobre los objetivos siguientes, que por otro lado constituyen la preocupación principal de las autoridades y gestores universitarios:

- aumentar el logro individual

- reducir las tasas de deserción escolar

- mantener la motivación del estudiante

- aumentar la efectividad del docente 
A esto habría que añadir obviamente lo que se supone objetivo implícito de las universidades: Aumentar la eficiencia de los aprendizajes obtenidos por los alumnos más allá del límite actual, superando barreras y dificultades que de otra forma no desaparecerían.

Pero no solamente están los beneficios que se derivan del uso de los entornos inteligentes para la mejora del rendimiento educativo que directamente proporciona la ayuda pedagógica. Hay otros beneficios que podríamos resumir en los cuatro ítems siguientes

La Educación Superior (ES) en nuestro entorno está en una posición ideal para preparar a los estudiantes para el mundo de la Inteligencia Artificial

Hay muchos nichos profesionales emergentes bajo el paraguas de la IA. Tradicionalmente la Universidad ha provisto de capacitación para los nuevos roles profesionales, máxime estos tan cercanos a ciencias y desarrollos teóricos básicos. En primer lugar, hay un nuevo rol para que la Educación Superior dote de equipamiento a los graduados para que trabajen efectivamente junto con sistemas artificialmente inteligentes.

Por lo tanto, los profesores universitarios deben garantizar, no solo para las titulaciones nuevas, sino también para las que tienen dominios de actuación tradicionales, que los estudiantes ingresen al lugar de trabajo con una comprensión de las competencias y de las limitaciones de Al, y la capacidad de continuar aprendiendo a medida que Al desarrolla y continúa cambiando los roles y las expectativas del lugar de trabajo.

La universidad no puede quedar al margen de esta oportunidad. Conlleva un riesgo similar al que las ingenierías no hubiesen adoptado los temas concernientes a los nuevos medios de transporte o los nuevos materiales como objetivos de capacitación y de investigación.

La IA puede ayudar a resolver grandes desafíos que la ES tiene en la Sociedad del Conocimiento, particularmente los que plantean las innovaciones disruptivas

En el terreno estratégico de la política universitaria, la Inteligencia Artificial puede proporcionar parte de la solución a los grandes desafíos con los que habitualmente se enfrenta la toma de decisiones de las universidades. Por ejemplo, el proceso de la enorme cantidad de datos educativos, cada vez mayor, sobre estudiantes. Datos que tienen disponibles las universidades.

Numerosos trabajos (Parr, February 28, 2013) han investigado los patrones sobre la actividad de los estudiantes, sobre datos que rutinariamente son registrados y analizados por las universidades usando "análisis de patrones de aprendizaje". Hasta ahora, estos análisis se usan principalmente para predecir qué estudiantes podrían fallar o abandonar. Pero, como hemos visto, el potencial principal es en el apoyo a la tutoría, que a partir del análisis de los procesos de aprendizaje de los alumnos individualmente, proporcione intervenciones oportunas en tiempo real para ayudarles.

La investigación que se realice en la universidad sobre IA, puede ser una parte clave y estratégica de la revolución de la IA en España, o en el país y en la sociedad, en particular, donde se desarrolle. En particular en el ámbito de la Educación 
Los investigadores y los educadores de la universidad son las personas y poseen los recursos de conocimiento clave para ayudar al país donde se ubiquen y a su economía a desarrollar el tipo correcto de sistemas de inteligencia artificial en los distintos ámbitos y servicios pero también en particular para su uso en educación, y debe hacerse sin demora.

\section{Las universidades están ubicadas en el contexto correcto para investigar la IA}

El contexto es una variable clave para la adquisición del conocimiento y es por tanto un factor básico en la efectividad de la educación. Los partidarios del aprendizaje situado señalan que es el factor más importante y algunas universidades de excelencia como Cambridge u Oxford así lo estiman.

El contexto, en este sentido, se puede definir como la combinación de personas, entornos, conocimiento, tecnología y recursos que constituyen una parte esencial de las interacciones de cada estudiante con el resto y con el mundo real. Son variables relevantes que intervienen en el éxito educativo de cada estudiante. Los desarrolladores de la IA deben conocer y trabajar por tanto el modelado contextual que permita a las herramientas de IA determinar cómo y por qué lo que funciona en una universidad no funciona en otra. $Y$ no trasplantar esquemas $y$ patrones de forma mecánica

La educación superior debe enfrentarse pues al desafío de la investigación de modelado contextual.

Resumiendo, la Inteligencia Artificial tiene el potencial de aportar grandes beneficios a la universidad. Sin embargo, solo cosecharemos estos beneficios si desarrollamos y usamos la IA como una innovación, es decir de una forma eficiente (Linden \& Fenn, 2003).

\section{El marco teórico y la pedagogía de un entorno de aprendizaje inteligente.}

Tras ver qué es el aprendizaje inteligente y los entornos inteligentes de aprendizaje, y cuáles son las ventajas de la IA para la universidad, es pertinente plantearse qué marco es necesario para la adopción eficiente de estas posibilidades, cuáles son las pedagogías que más partido pueden sacar de ellas. Hay que construir un marco educativo.

Algunos teóricos, haciendo una síntesis, sostienen que la instrucción se reduce estrictamente a un problema de entrega: Entrega de ayuda y entrega de recursos (Merrill, 2002 y 2009; Reigeluth, 1999; Clark, 2003; Clark and Mayer, 2003). Y que las estrategias de enseñanza consisten exclusivamente en encontrar el momento y el lugar de la entrega, en función del estudiante, de su situación respecto del aprendizaje y de su contexto. En este sentido una pedagogía apropiada es la que use la inteligencia artificial para determinar qué se entrega, en qué momento se hace y cómo se hace. En determinar esto último tienen bastante que decir los principios clásicos y universales del aprendizaje. O lo que es lo mismo: saber y utilizar lo que conocemos acerca de cómo los individuos aprenden. 
Para ello es básico conocer al menos, y aplicar a este nuevo contexto, los citados "principales principios del aprendizaje" estudiados y enunciados de forma sistemática por David Merrill (Merrill, 2002 y 2009) y las "condiciones del aprendizaje" investigadas y enunciadas por Gagné (1965 y 1985) y Gagné \& Briggs (1974), así como los elementos de diseño instruccional de Reigeluth (1999) y de Fink (2003).

Estos son principios universales también operativos en los entornos inteligentes de aprendizaje, pero además hacen falta incorporar innovaciones pedagógicas específicas para estos entornos. Existe una necesidad de incorporar innovaciones pedagogías para apoyar los nuevos requisitos de aprendizaje. Así es percibido por ejemplo por Chen, Cheng y Chew (2016).

En lo que sigue veremos muy sucintamente algunos ejemplos de innovaciones pedagógicas que permiten identificar algunas diferencias fundamentales que establecen los entornos de aprendizaje inteligentes de forma divergente a los enfoques tradicionales.

\section{Las microinteracciones sociales y el conocimiento generado a partir de ellas. El aprendizaje oportunista (Bopportunistic learning)}

Además de las situaciones de aprendizaje formales ahora hay otras. El conocimiento en entornos de aprendizaje inteligentes no se limita a una reproducción mimética de actividades convencionales, al igual que un email no es una carta, ni como se ha dicho son una virtualización (reproducción idéntica como en un espejo digital) de ellas.

Ahora se empieza a aceptar que el aprendizaje puede suceder y sucede en cualquier entorno, y que la interacción y la conversación cuando los estudiantes se involucran produce insights de aprendizaje (Dabbagh y Kitsantas 2012). No es un aprendizaje inteligente, es lo que se conoce como aprendizaje social. Pero veamos qué sucede después. Cuando los alumnos se implican en los medios sociales, como en la navegación por los posts de Facebook, la lectura de tuits en Twitter, o simplemente al tener una conversación con alguien en Skype o WhatsApp, se producen momentos de aprendizaje. Ahora el reto para la nueva pedagogía, y esto sí que forma parte de las bases de la pedagogía inteligente, la que ayuda al aprendizaje inteligente, es identificar esos momentos de aprendizaje --- Bopportunistic learning--- (Kinshuk 2014) mediante el análisis de las micro-interacciones sociales. Y a partir de ello ver cómo son estas píldoras ${ }^{2}$ de conocimiento ---knowledge nuggets--- que se generan y cómo se puede producir/favorecer su integración con el conocimiento previamente adquirido por el alumno. Es decir la inserción en sus andamiajes cognitivos.

\section{La evaluación del conocimiento generado a partir de micro-interacciones sociales}

Lo que hemos visto antes, la integración efectiva de los conocimientos generados mediante las micro-interacciones sociales, también plantea unas necesidades nuevas en la evaluación: Las prácticas de evaluación también necesitan reconsideración. Algo parecido sucede con el

\footnotetext{
${ }^{2}$ La traducción literal de nugget es pepita, semilla de fruta aunque también en inglés este término sirve para nombrar a un preparado culinario en forma de pequeñas albóndigas. Esta expresión en inglés quizá haga más divertido el concepto. Sin embargo en español parece más aconsejable utilizar la palabra píldora, que en castellano hace referencia a pequeñas dosis, y que tiene más sentido hablando de micro aprendizajes.
} 
análisis de la relevancia de las interacciones en los foros, y lo que hemos hecho en el curso investigativo de la Universidad de Murcia que después comentaremos con más extensión. Obviamente los métodos de evaluación formales, como de dos horas de exámenes finales en una sala de examen con papel y lápiz, o responder cuestionarios en el ordenador, no proporcionan medios adecuados para evaluar las knowledge nuggets que los alumnos han adquirido durante sus interacciones sociales, o la integración de esas píldoras de conocimiento en su andamiaje cognitivo atribuyéndoles sentido (Kinshuk 2015). Se necesitan pues métodos nuevos de evaluación para analizar los fenómenos de adquisición del conocimiento debido a las interacciones sociales, así como el impacto que tienen en el nivel de competencia del alumno individual. La investigación se espera que nos dé resultados acerca de análisis necesario para la evaluación y nos indique métodos de identificar patrones en el comportamiento del alumno que se puedan extrapolar desde el nivel micro.

Hasta aquí ejemplos de situaciones emergentes que requieren pedagogías propias

Por otra parte hay un espacio pedagógico que no es la aplicación de los principios universales del aprendizaje a los entornos inteligentes ni algo tan distinto e innovador como la pedagogía para las pildoras de aprendizaje.

Nos referimos a ámbitos nuevos propios de los entornos tecnológicos pero donde hasta ahora la intervención había sido exclusivamente humana.

Vamos a aportar, para ilustrar ese tercer nivel de ámbitos pedagógicos donde puede intervenir el proceso inteligente en alguna medida, un caso desarrollado en la Universidad de Alcalá, en el que ha participado el autor. Se trata de una experiencia de curso abierto online que, en su cuarta edición desde 2014, se está desarrollando en dicha universidad, apoyado por el Departamento de Computación y el Servicio de Extensión Universitaria. En él ha participado unos 1500 inscritos en los cuatro años y en las tres capas de que consta.

La Universidad de Alcalá es una de las más antiguas de Europa, sin embargo ello no impide que apueste por las modalidades docentes más innovadoras y disruptivas, como son la educación abierta, y dentro de ella los cursos dual layer (Siemens, 2014 May; Crosslin, 2014 May), con metodología de Mastery Learning (Bloom, 1984) y el diseño instruccional de los cursos abiertos online. Se hace en la plataforma para MOOC de BlackBoard: Open Edition.

"El diseño instruccional de los cursos abiertos on-line" ${ }^{3}$ está destinado a profesores y diseñadores de estudios abiertos online Se trata de un curso abierto, personalizado, y de acceso escalable en la metodología docente y en la ayuda pedagógica. Es decir no es un MOOC al uso, tiene unos rasgos propios, los de un nuevo tipo de cursos. Toda la información se difunde en un blog ${ }^{4}$

Hasta ahora son nueve las universidades e instituciones que han incorporado, en la organización y en la práctica de la docencia, las habilidades sobre Diseño Instruccional

\footnotetext{
${ }^{3}$ El diseño instruccional de los cursos abiertos on-line https://openeducation.blackboard.com/mooccatalog/courseDetails/view?course_id=_1684_1

${ }^{4}$ http://cursosabiertos.blogspot.com.es/
} 
obtenidas por profesores y gestores de dichas universidades e instituciones en este curso $^{5}$ : Universidad Técnica Particular de Loja (Ecuador), Universidad de Valladolid (España), Universidad de Quilmes (Argentina), Universidad de Guadalajara (México), Universidad de Alcalá (Máster de Ciberseguridad y Análisis de datos), Universidad Autónoma Intercultural de Sinaloa (México), CRESUR Chiapas (México), Natanael SL y Hewlett Packard (Estas dos en el Máster de Ciberseguridad y Análisis de datos de la Universidad de Alcalá).

En el desarrollo del curso, al principio, la analítica nos dio los siguientes datos:

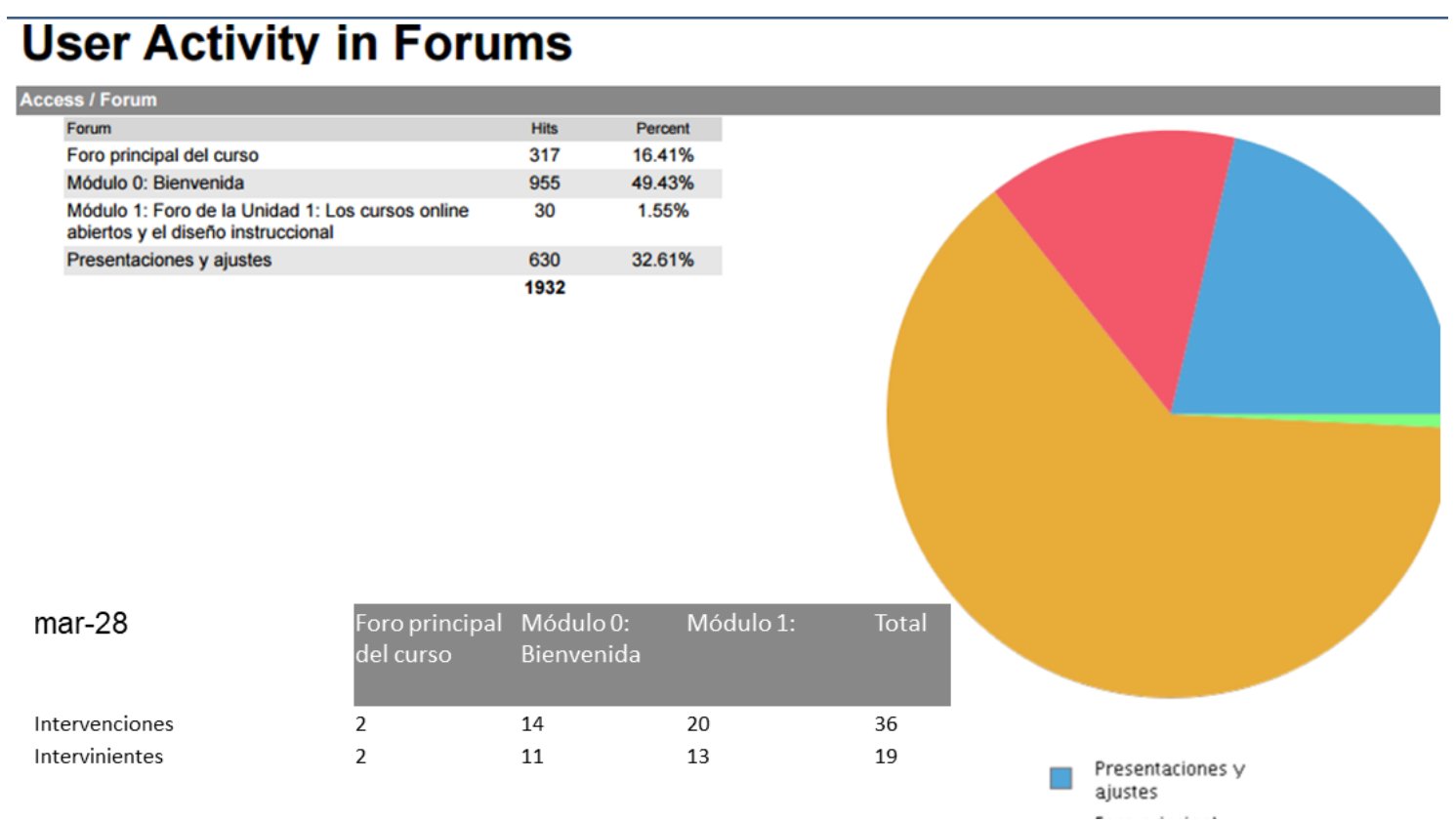

Fig. 4

Había muchos accesos a los foros, casi dos mil insights, pero la actividad era escasa 36 intervenciones de 19 intervinientes. Nada sabíamos a partir de la analítica de la relevancia de las intervenciones. Eso lo sabían los Teaching Assistants (TA). Tomamos la decisión de pautar y analizar la relevancia de las intervenciones, adjudicándoles valor para el logro. El resultado fue un significativo aumento de las intervenciones, en número y también de accesos:

\footnotetext{
${ }^{5}$ Para figurar en esta lista ha sido necesario que esas habilidades hayan redundado en un cambio relevante en enseñanzas de grado, de postgrado o en estudios propios en línea.
} 


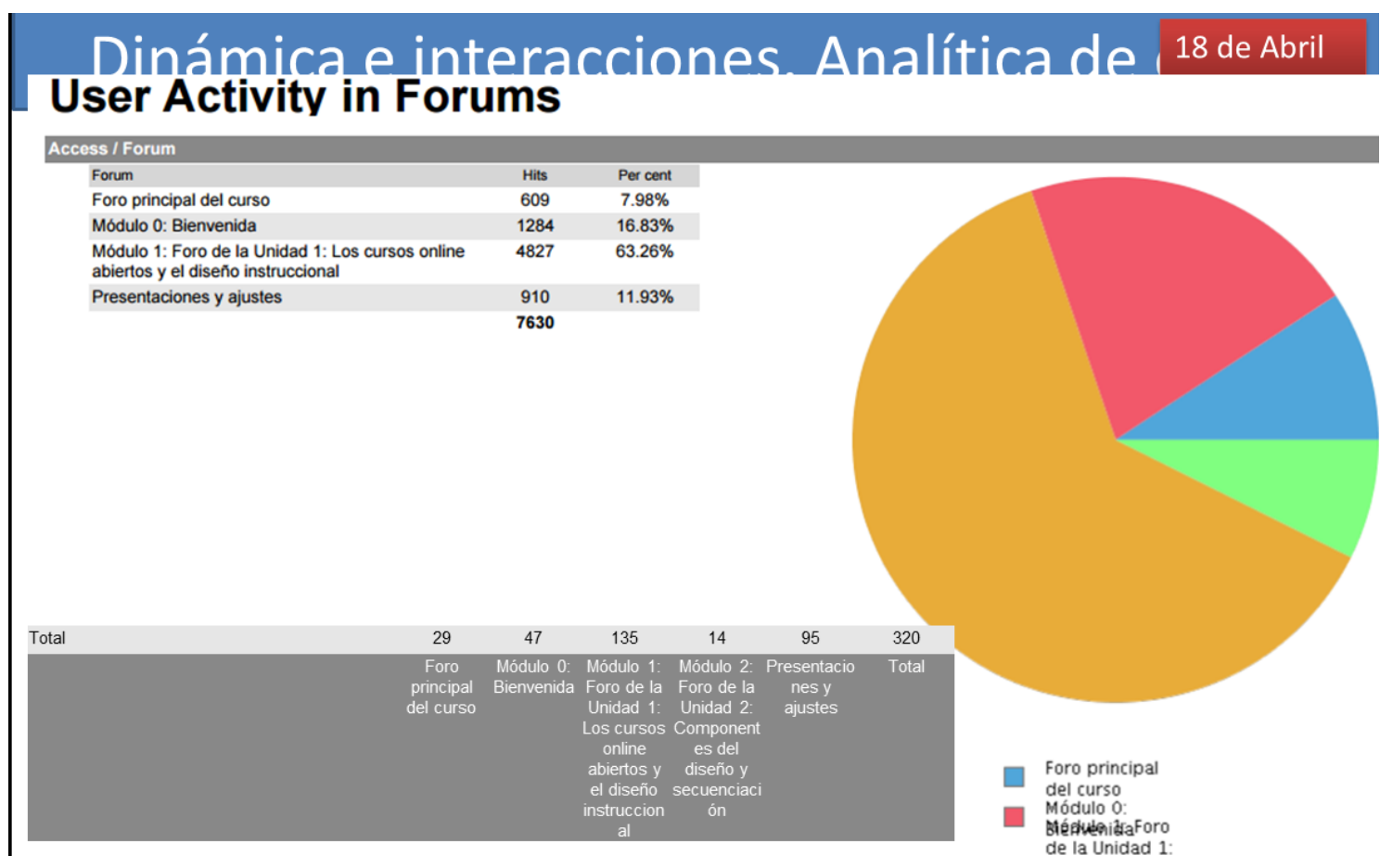

Fig. 5

Del 28 de marzo al 18 de abril pasaron de 36 a 320.

Este tipo de evaluación formativa es fácilmente implementable en un algoritmo. Casi sólo son un par de instrucciones condicionales.

Un aspecto más interesante es el de la relevancia en las intervenciones. En un aprendizaje de tipo conceptual, como era el de este módulo eso adquiere una importancia clave en nuestra metodología, de mastery learning. En el curso lo resolvíamos, en este punto, si el participante contribuía con dos intervenciones relevantes en el foro. La cuestión era qué es la "relevancia". Porque el sistema adjudicaba automáticamente el logro cuando detectaba dos intervenciones valoradas por los TA como relevantes.

A los participantes en la guía didáctica del módulo se le daban instrucciones precisas (volcado de pantalla de la plataforma Open Education): 


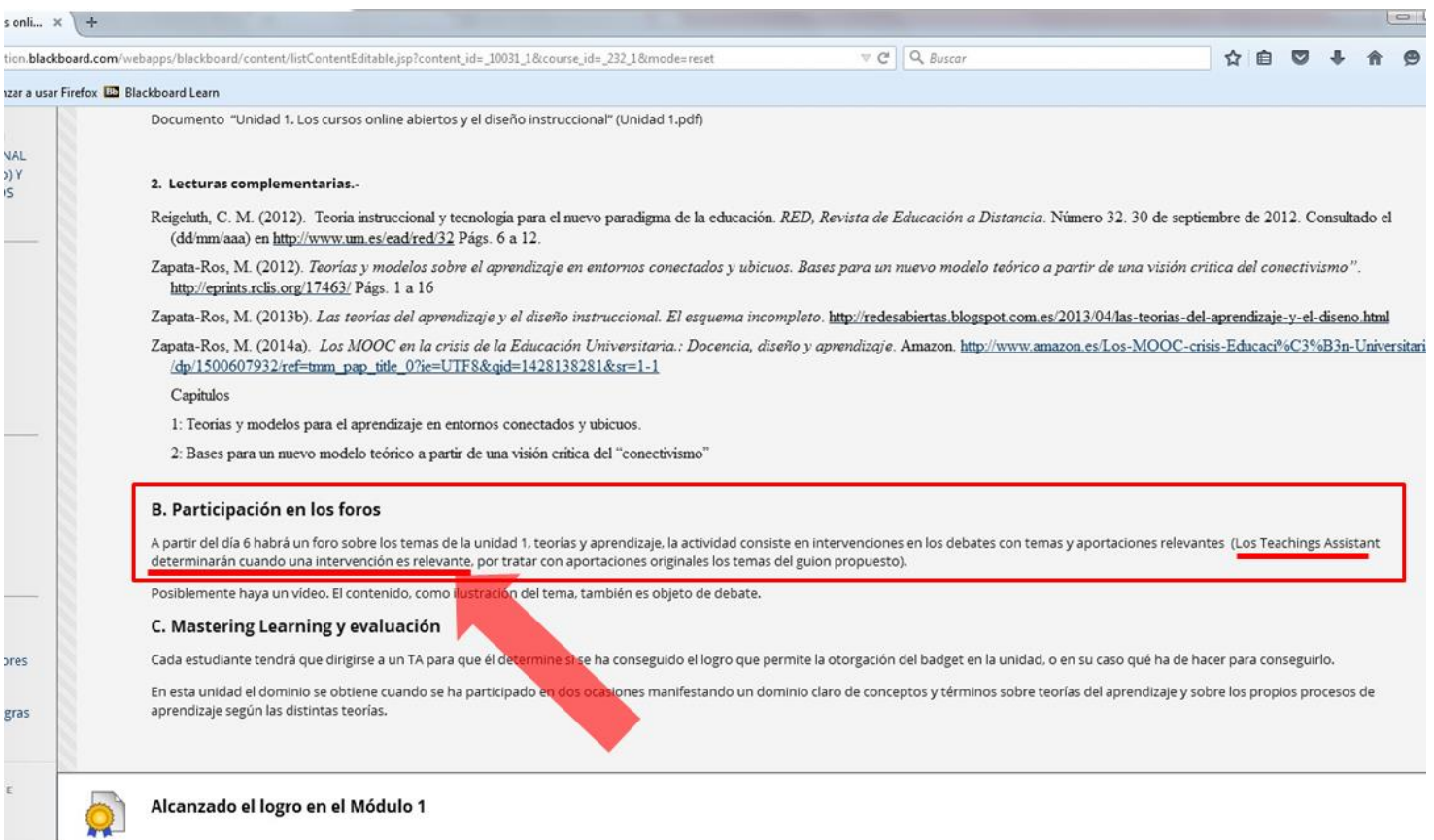

Fig. 6

Ese análisis era efectuado mediante lectura, evaluación, notas al participante y retroalimentación por el TA, que finalmente acreditaba que el alumno había obtenido el logro:

\section{Análisis de la relevancia en foros y logros}

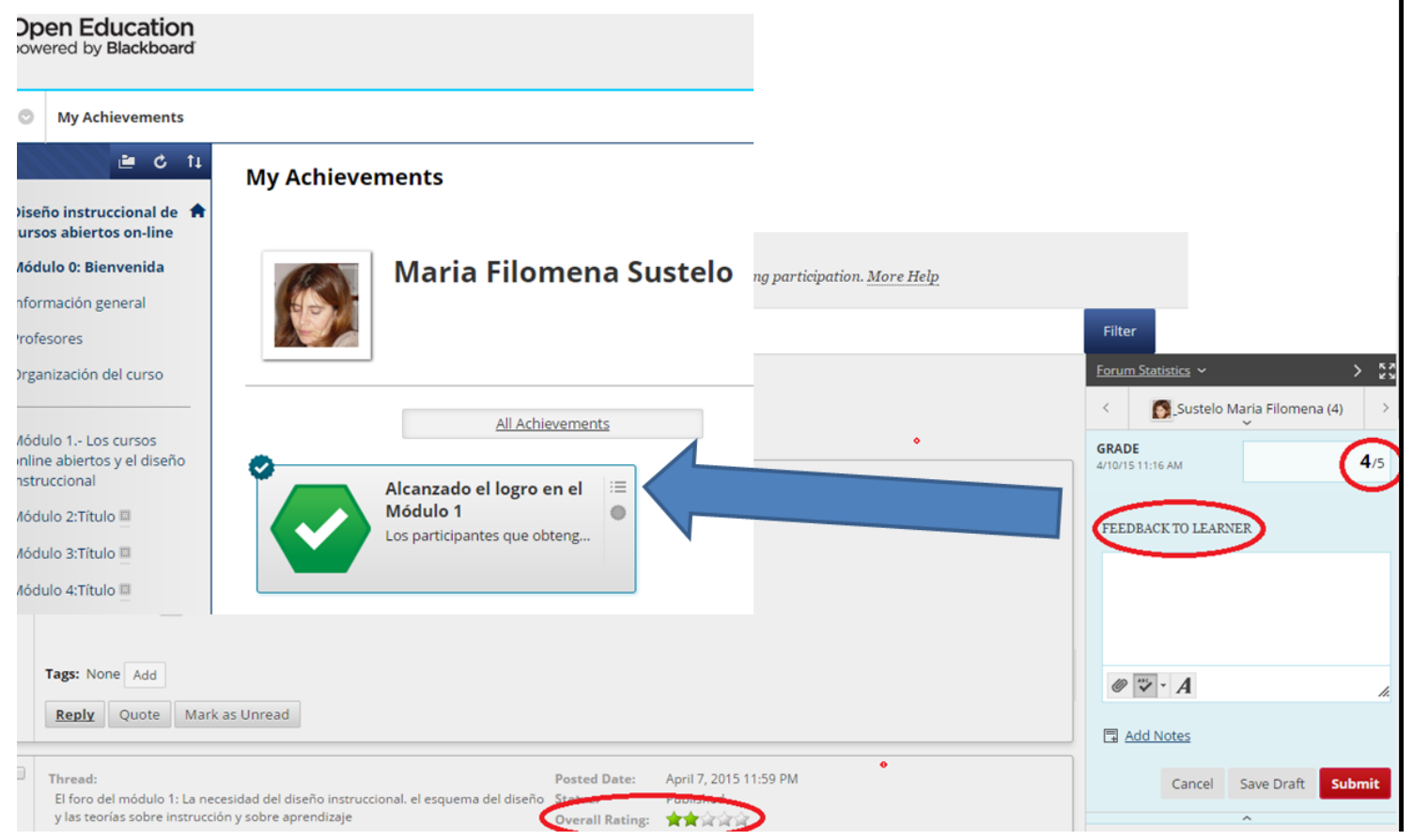

Fig. 7 
El criterio para que el TA adjudicara la relevancia era una reelaboración de lo analizado en el trabajo de Gagné "Las condiciones del aprendizaje" (Gagné, 1965 y 1985) (Driscoll, 1994 y 2013), a partir las condiciones que establece para que el aprendizaje sea efectivo, en particular de lo que establece para los nueve niveles de aprendizaje (Gagne's Nine Levels of Learning) ${ }^{6}$ :

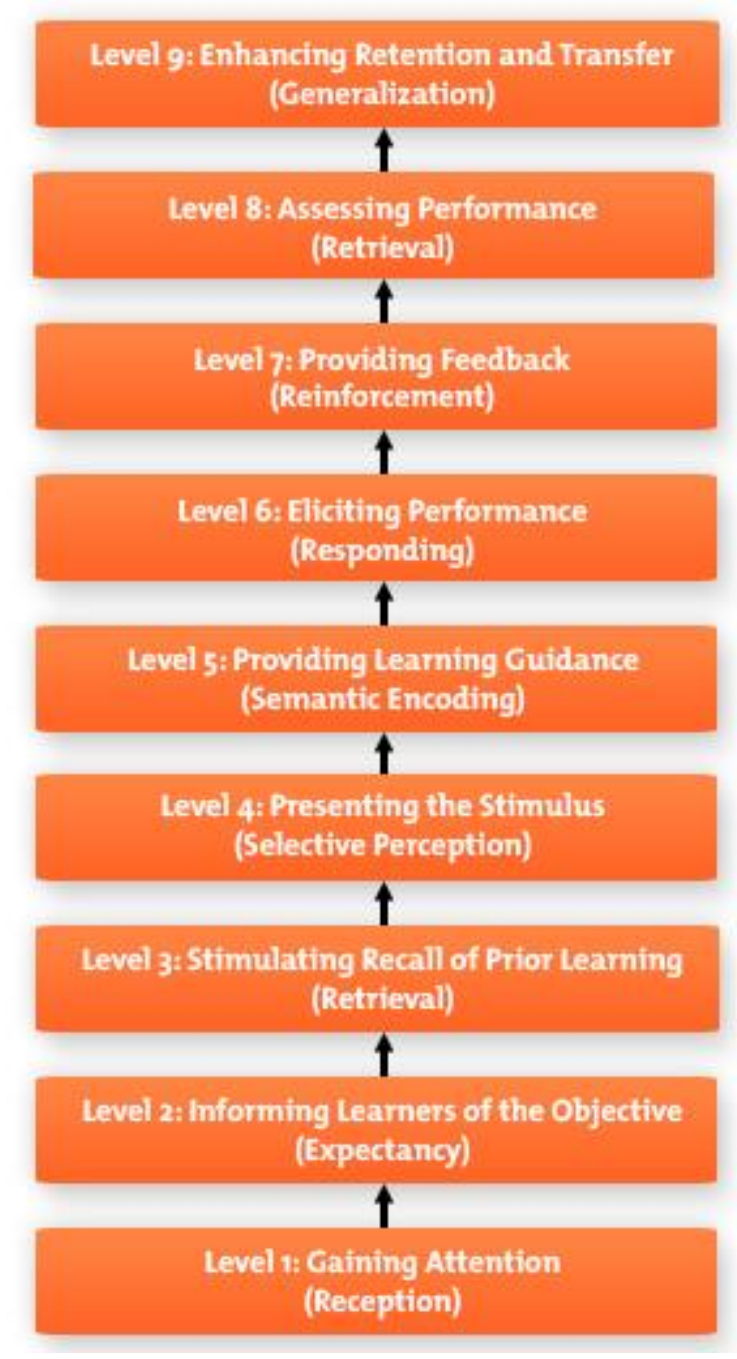

Fig. 8

Y una reelaboración, cuya justificación teórica expondremos en un próximo trabajo, de tal forma que interpretándolos con la ayuda de lo elaborado por Bloom (1984) para el problema de dos sigmas, y la metodología docente de Mastery Learning, el dominio del aprendizaje supone al menos las siguientes características de lo aprendido:

1. Comprensión

2. Adquisición y atribución de sentido

3. Ejecución autónoma

4. Transferencia

\footnotetext{
${ }^{6}$ Sintesis de Gagné's Conditions of Learning para habilidades intelectuales y estrategias cognitivas.
} 
En el caso que nos ocupa todo esto se concretaba en el siguiente procedimiento para utilizar por los TA y que era conocido por los participantes, en la determinación del grado de relevancia de las contribuciones al foro en este caso:

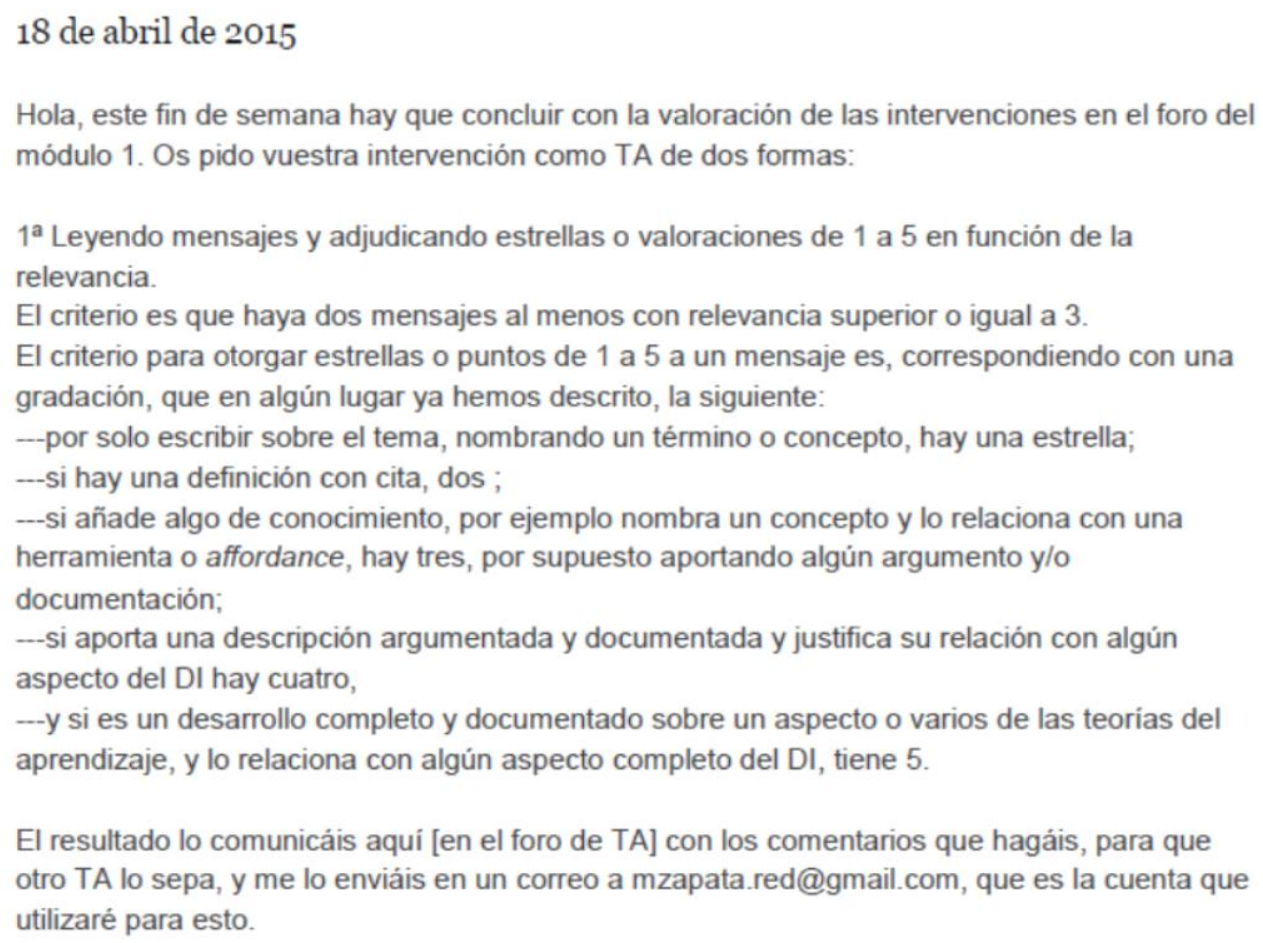

Fig. 9

La cuestión en este punto es trasladar los criterios señalados, que utilizaban los TA, a procedimientos inteligentes a aplicar en un sistema inteligente. Cómo se implementa por ejemplo, "escribir sobre el tema nombrando un término o un concepto", se supone que en un contexto con sentido lógico y con relación al dominio disciplinar, o si "nombra un concepto y lo relaciona con una affordance", también en un contexto textual con sentido. Etc. para que el sistema lo detecte en una contribución al foro que evalúe con un grado de relevancia. 
Aquí dejamos la cuestión, en esta modalidad de líneas de desarrollo, a la espera de propuestas interesantes o de experiencias ya desarrolladas en esta dirección.

\section{Ejemplos y experiencias}

\section{BI0100 de Global Freshman Academy}

Entre los varios ejemplos significativos que hay de la adopción de la IA para organizar el aprendizaje y la asistencia a los alumnos, podemos destacar el de la Arizona State University, particularmente el caso de la Global Freshman Academy apoyada por la plataforma software de Inteligencia Artificial ALEKS de McGraw-Hill Education.

Este caso ha sido señalado tanto por Clark (February 19, 2016) en sus post de plan B que hacen un extracto de su informe, como por el informe de la CRUE (Delgado et al, 2017))

Las conclusiones son que la IA en general, y los sistemas de aprendizaje adaptativo en particular, tendrán un enorme efecto a largo plazo en la mejora de la calidad de la enseñanza demostrada en los logros, en la eficiencia del aprendizaje, el rendimiento del alumno y el descenso del abandono escolar.

Esto fue confirmado por los resultados de los cursos realizados en la Universidad Estatal de Arizona en otoño de 2015 presentados en Educause Learning Initiative en San Antonio en febrero de 2016. Los datos y gráficos están obtenidos de Donald Clark (February 19, 2016)

La referencia es el curso, Biology 100 (BIO100) ${ }^{7}$, en modalidad de blended learning En él se hizo el informe. Se llevó a cabo en la plataforma CogBooks ${ }^{8}$. Está descrito por Clark (February 19, 2016): Las actividades las llevaron a cabo en la plataforma y luego el trabajo grupal y las dudas se llevaron a clase en modalidad flipped classroom.

El informe se centra en los objetivos del curso, como experimento de sistema de enseñanza mejorado con tecnología:

- aumentar el logro

- reducir las tasas de deserción escolar

- mantener la motivación del estudiante

- aumentar la efectividad docente

Respecto del primero, mejorar los resultados del aprendizaje esta gráfica con expresión del tamaño de la muestra es elocuente:

\footnotetext{
${ }^{7}$ https://www.coursehero.com/sitemap/schools/168-ASU/courses/639255-BIO100/

${ }^{8} \mathrm{https}: / /$ www.cogbooks.com/biology/
} 


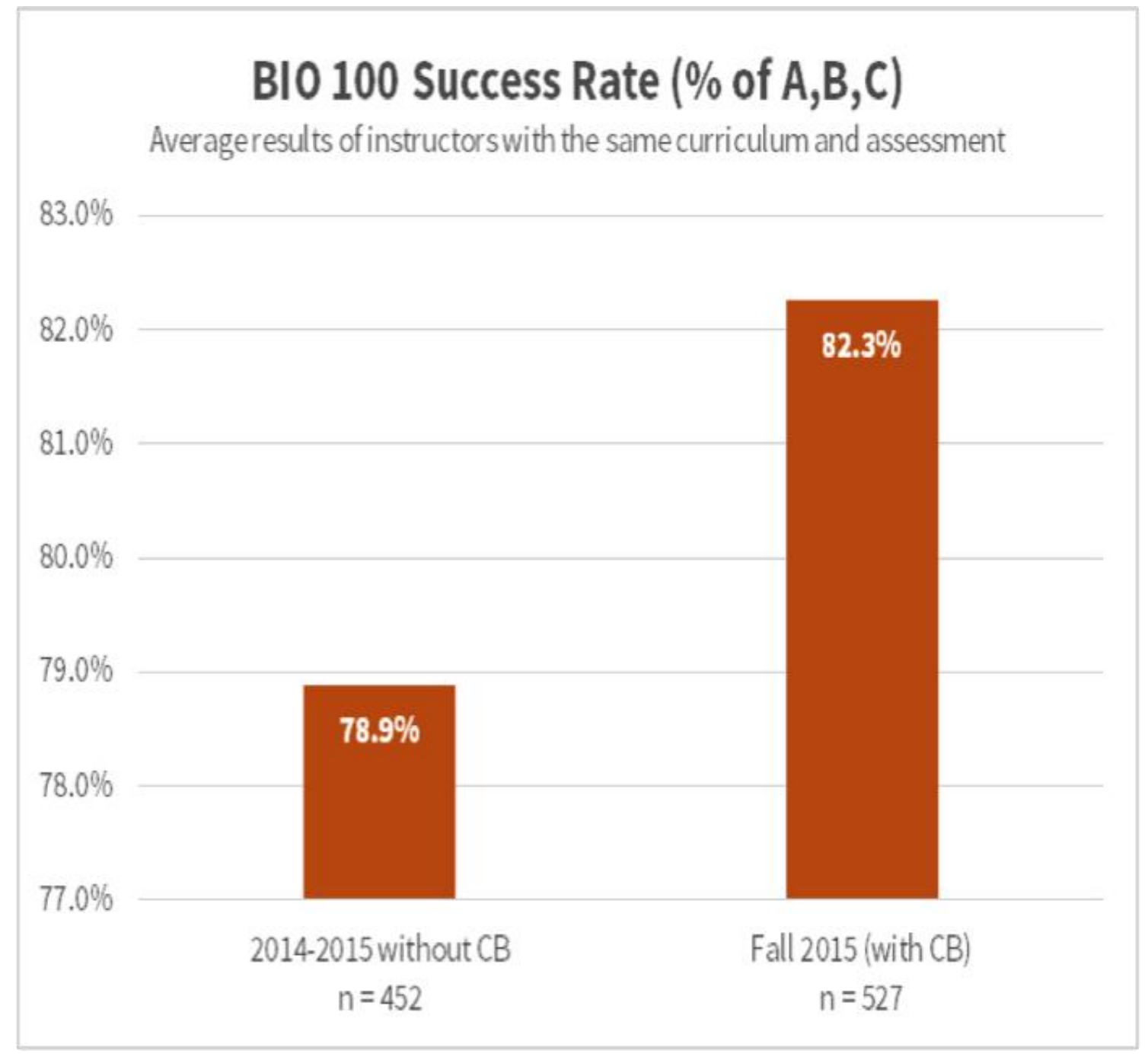

Fig. 10

Respecto del abandono 


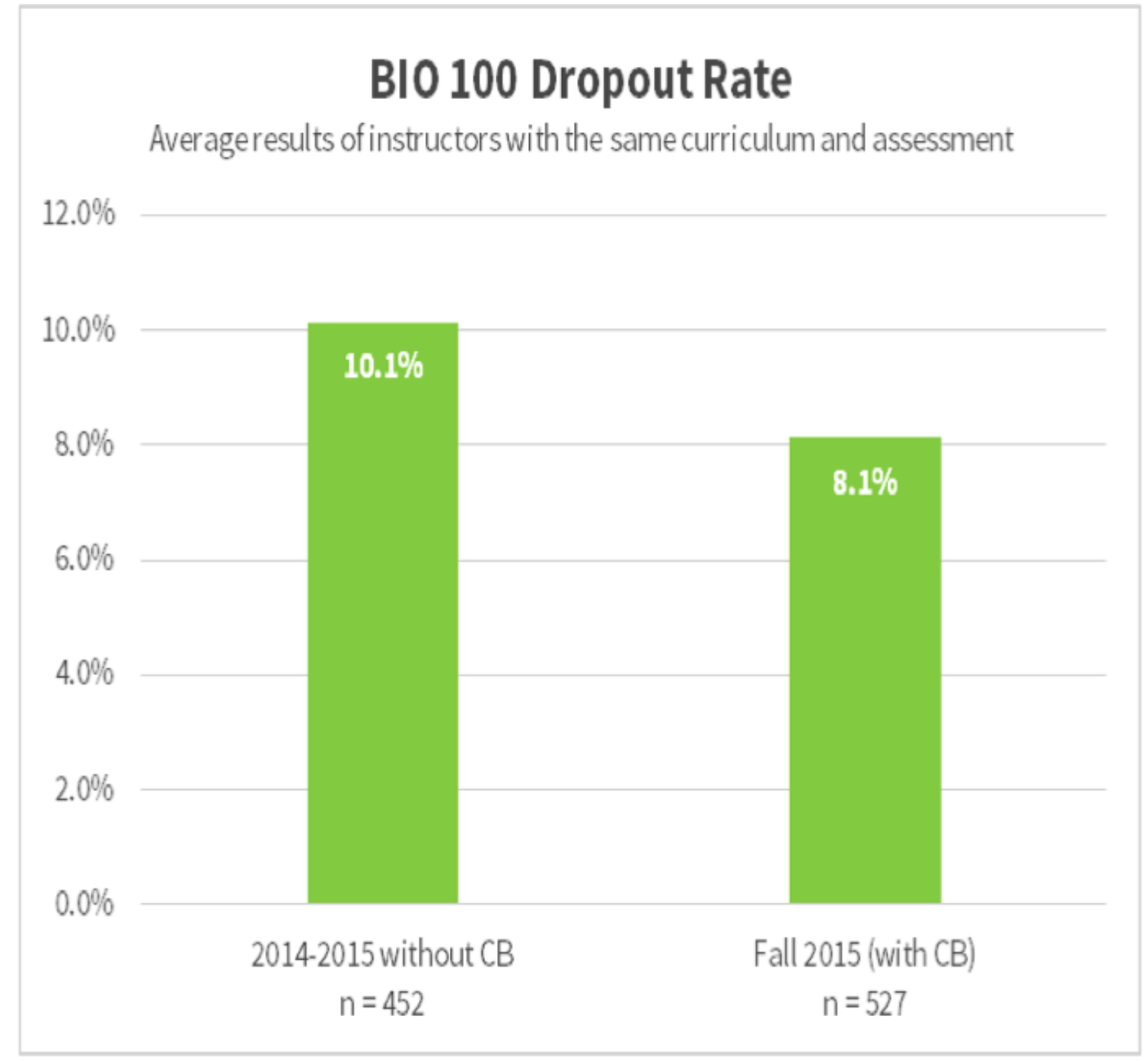

Fig.11

Se reduce en la misma institución en un dos por ciento. Pero esto no es lo importante, lo importante es comparar con esta modalidad de enseñanza en estudios similares que en EE UU está entre el $41 \%$ y el $45 \%$ de los que se matriculan en universidades estadounidenses. Lo cual es dramático si consideramos que después tienen que hacer frente a la deuda, que en estos tipos de estudiantes es en EE UU de 1,3 millones de dólares y el hecho de que estos estudiantes abandonaron, pero todavía cargan con la carga de esa deuda, este es un nivel catastrófico de fracaso. En el Reino Unido la tasa de abandono es de $16 \%$.

Los tableros de los profesores no son muy distintos de los que hemos utilizado en Open Education, y que hemos descrito en un apartado anterior 


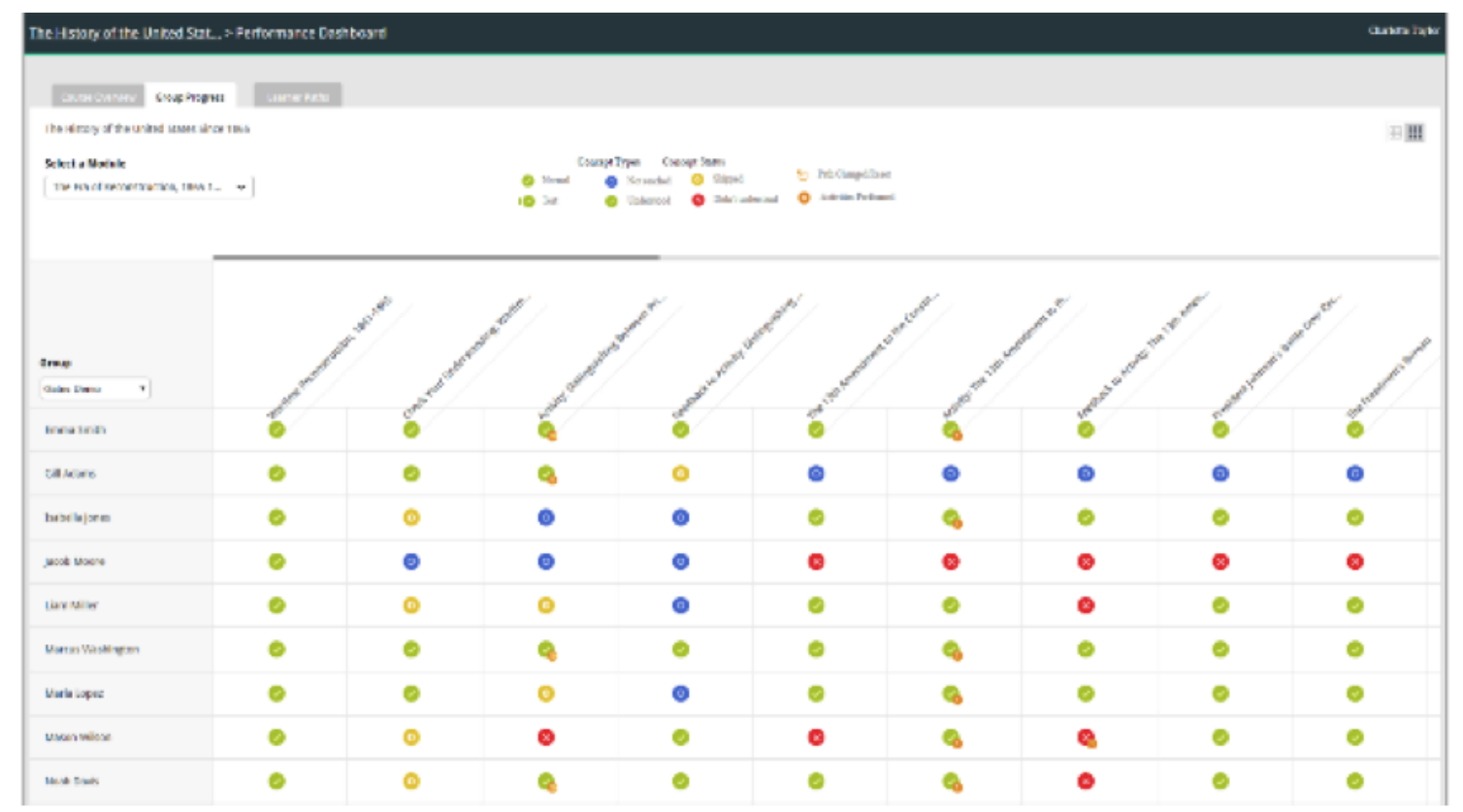

Fig. 12

Diferentes paneles de control brindan información, en tiempo real, del rendimiento de los estudiantes. Esto le permite al instructor ayudar a los necesitados.

La realidad es que según Clark el sistema promete en este punto una mejora continua, "muy necesaria en educación". No ofrece evidencias de una aportación concreta de la IA: "Podríamos mirar un enfoque que no solo mejore el desempeño de los docentes sino también el del sistema en sí, con la consecuencia de una mejora continua en el rendimiento, la deserción y la motivación en los estudiantes".

No creemos que los selft report studies sean un instrumento adecuado para la investigación en el ámbito del aprendizaje y de la instrucción. Como bien hemos justificado en otras ocasiones no son un elemento significativo del rendimiento del aprendizaje ni de la calidad de la docencia. En algunos casos la correlación es inversa. No obstante hay que señalar que en este caso el informe dice "que los estudiantes quieren más" 


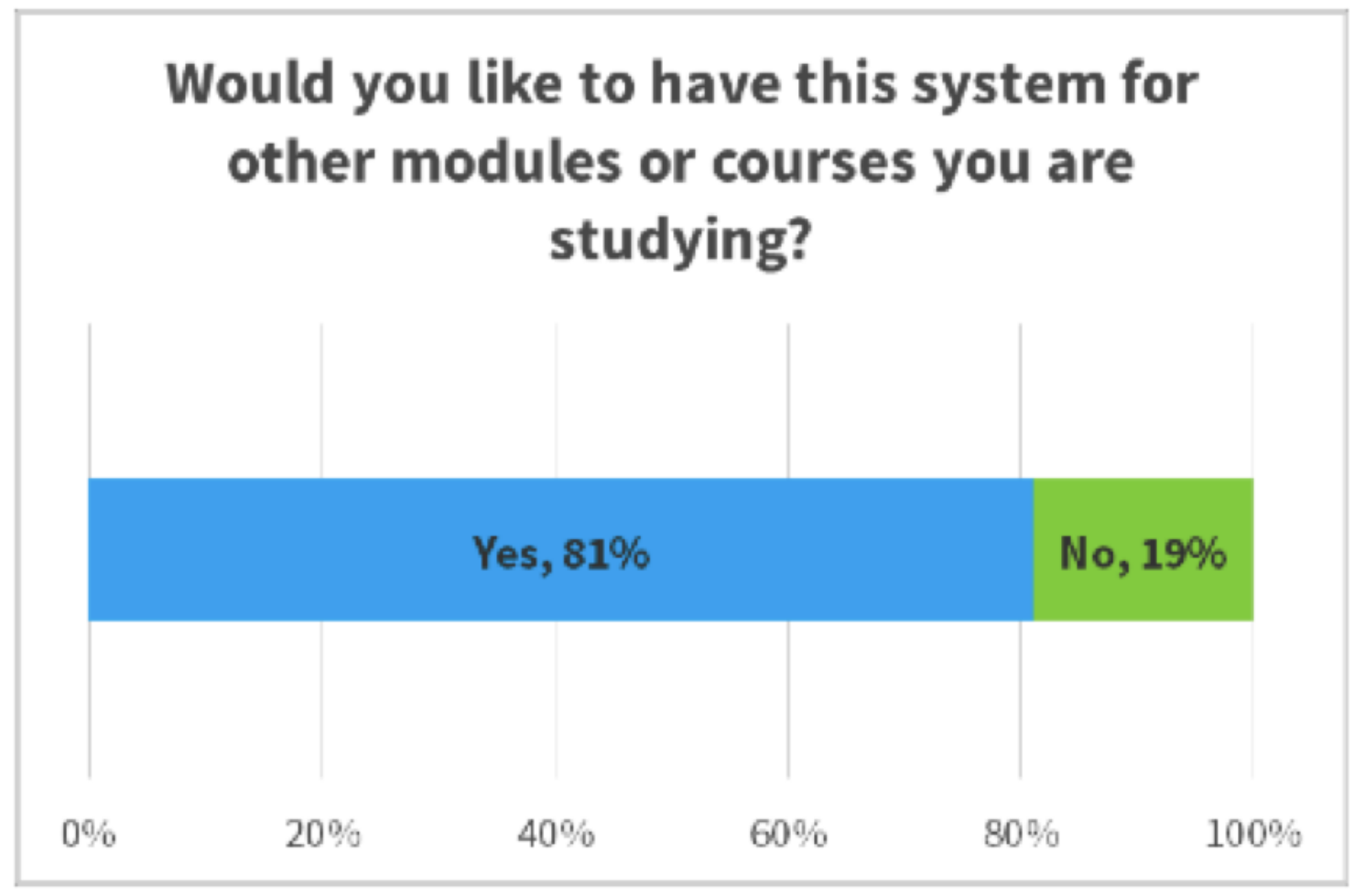

Fig. 13

Más del $80 \%$ de los estudiantes en esta primera experiencia de un curso adaptativo, dijeron que querían utilizar este enfoque en otros módulos y cursos.

Sin embargo no hemos obtenido una información técnica (sobre la metodología docente y de apoyo al alumno) detallada. Tampoco nos la ha dado Clark.

Lo más aproximado es la información que da la propia plataforma CogBook en pantallas como ésta:

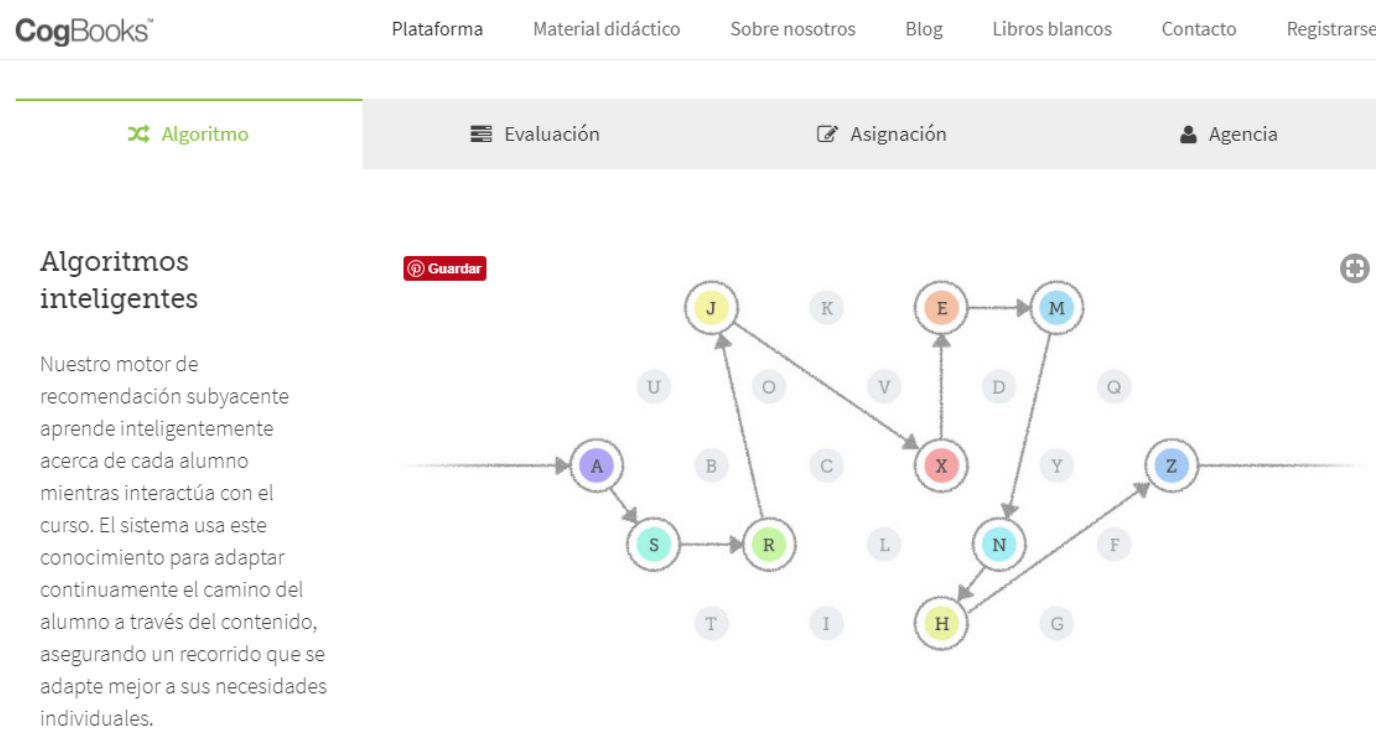

Fig.14 
Lo más aproximado es la descripción que hace Clark (February 19, 2016) en el párrafo siguiente:

"Una de las dificultades en los sistemas adaptables, impulsados por Al, es la creación de contenido utilizable. Por contenido, me refiero a materiales de aprendizaje, estructuras, elementos de evaluación, etc. CogBooks ha creado un conjunto de herramientas que permiten a los instructores crear una red de contenido, trabajando desde objetivos. También se usa la ayuda automática con el diseño y con la conversión de contenidos. Una vez hecho esto, se crea una red compleja de contenido de aprendizaje a través de la cual los estudiantes circulan, cada estudiante mediante un camino diferente, dependiendo de su rendimiento continuo. El sistema es como un satélite, siempre trata de llevar a los estudiantes a su destino, incluso cuando se salen de curso."

También el propio Clark (JANUARY 13, 2016) ha descrito una taxonomía para los sistemas Al adaptativos (sistemas inteligentes) de apoyo al aprendizaje en base a la asistencia con cinco niveles:

\section{Nivel 1: Tecnológico}

Nivel 2: Asistido

Nivel 3: Analítico

Nivel 4: Híbrido

Nivel 5: Autónomo

Él sitúa la experiencia de CogBook en BIO100 en el nivel 4, sistema adaptativo híbrido, con mezcla de ayuda humana y de detección y de recomendación. Algo que guarda ciertas similitudes con lo que hemos descrito para el análisis de la relevancia en las contribuciones de los foros en el curso "El diseño intruccional de los cursos abiertos online", de la Universidad de Alcalá. Que actualmente estaría en el límite entre 3 y 4.

\section{Otras experiencias reales.-}

Otras experiencias actuales que hemos examinado, rastreando los enlaces de una presentación hecha a la CRUE para justificar el Informe Tendencias TIC 2018 (Delgado et al, 2017) han sido las dos siguientes:

Al en educación - The Genie of Deakin University.- En la universidad Deakin ${ }^{9}$, en Victoria, Australia, se ha estado experimentando con la plataforma Watson durante bastante tiempo

\footnotetext{
${ }^{9}$ https://medium.com/@Hubert.ai/ai-in-education-the-genie-of-deakins-universitye29fbcde27d1
} 
con el fin de servir a sus alumnos de una manera más personalizada. Desde 2015, los estudiantes han podido pedir consejo a Deakin Genie, con sede en Watson, sobre una amplia variedad de temas.

\section{Jill Watson: la primera asistente de enseñanza de IA de Georgia Tech ${ }^{10}$}

El equipo dirigido por Goel y Polepeddi, L. (2016) ha trabajado sobre el uso de sistemas basados en herramientas de respuesta y recomendación para responder a las preguntas de los estudiantes en los foros de su clase en línea de Inteligencia Artificial basada en el Conocimiento (KBAI).

La asistente ha sido nombrada Jill Watson, se basó en la plataforma Watson de IBM, que es quizás mejor conocida como la computadora que venció a dos campeones de Jeopardy. Jill se desarrolló específicamente para manejar la gran cantidad de publicaciones en el foro por parte de los estudiantes inscriptos en un curso en línea que es un requisito para obtener el título de maestría en ciencias en el programa de informática de Georgia Tech.

\section{Discusión.- Cuestiones pendientes y líneas de investigación abiertas sobre el aprendizaje inteligente}

Hasta aquí la tesis que hemos defendido es que el aprendizaje inteligente en la Educación Superior es un nuevo concepto, una nueva ratio, del aprendizaje potenciado por la tecnología y de la enseñanza abierta universitaria. No se reduce pues a integrar los criterios y funciones de los sistemas de tutoría inteligente, de los sistemas de aprendizaje ubicuo conscientes del contexto, o de los propios sistemas adaptativos en un marco ya existente. Es una evolución de lo anterior con naturaleza propia, una fase distinta en una línea de progreso que ya existe; En ese contexto se plantean los siguientes temas de investigación para reconocer la presencia diferenciada de la concepción de "aprendizaje inteligente":

1.

Desarrollo de marcos de implementación tecnológica de entornos de aprendizaje inteligentes que alojen, con las funciones y affordances señaladas (apoyo en el aprendizaje y en la entrega pertinente y relevante de contenidos), el resultado del rápido avance de las tecnologías digitales (por ejemplo, realidad aumentada, visión artificial, reconocimiento de voz, tecnologías portátiles e inteligentes) y de las herramientas analíticas, por ejemplo: análisis de aprendizaje y tecnologías de conciencia social (social-awareness technologies) (Hwang, G. J. (2014)).

\footnotetext{
${ }^{10}$ https://pe.gatech.edu/blog/meet-jill-watson-georgia-techs-first-ai-teaching-assistant
} 
2.

Desarrollo de marcos del tipo anterior que ofrezcan posibilidades diversas de implementación de entornos de aprendizaje inteligentes basados con diferentes propósitos educativos y desde diferentes perspectivas de las teorías pedagógicas y del aprendizaje. Es por tanto un tema prioritario y un reto proponer marcos de implementación tecnológica e instruccional de entornos de aprendizaje inteligente con estas tecnologías emergentes.

3.

Reinterpretación y examen de las teorías pedagógicas y de las teorías sobre el aprendizaje existentes a la luz de lo que ofrecen los nuevos entornos de aprendizaje inteligente. Las nuevas formas de aprender nos demandan cómo organizar la instrucción en estos entornos. Hay un nuevo concepto de aprendizaje; por lo tanto, los investigadores y los docentes deberán proponer nuevas formas de enseñar, nuevas metodologías y estrategias docentes basadas en las teorías existentes, como el constructivismo, la teoría de la motivación, el modelo de aceptación de la tecnología, la teoría de la carga cognitiva o la teoría del diseño de entornos tecnológicos.

4.

Nuevas estrategias de aprendizaje y de evaluación para el aprendizaje inteligente.

5.

Ámbitos nuevos de aprendizaje que antes no eran asequibles a la tecnología. Por ejemplo la cinestesia.

Se espera que con los nuevos enfoques inteligentes y los nuevos conceptos de aprendizaje, se podrán poner en marcha investigaciones en nuevos dominios de aplicación, que ahora son difíciles de implementar en los entornos de aprendizaje existentes, y con los actuales y ya obsoletos LMS

6.

Rendimiento del aprendizaje por percepción y evaluación de la percepción. Se deben potenciar mecanismos de autorregulación y de determinación de la autoeficacia.

7.

Tipologías y patrones de comportamiento de aprendizaje y análisis de patrones de aprendizaje. Y más importante aún, las observaciones a largo plazo y el análisis de las conductas de aprendizaje podrían desarrollarse de manera que los investigadores puedan conocer más acerca de los impactos sociales, a través de la nueva tecnología educativa. 


\section{Conclusiones}

La presencia y el avance de las tecnologías de detección y de recomendación están ofreciendo grandes y determinantes oportunidades para desarrollar nuevos entornos de aprendizaje. Definitivamente el aprendizaje inteligente es un concepto extremadamente innovador, como en otros ámbitos, para desarrollar visiones y planteamientos distintos. En este caso para crear entornos de aprendizaje más potentes y útiles que los enfoques de aprendizaje ayudados por la tecnología ya existentes, mediante la incorporación de nuevas herramientas y nuevos criterios para el aprendizaje.

Todo ello es prometedor pero supone importantes desafíos que son claves para el desarrollo y la evolución de la nueva universidad que salga de las actuales disrupciones.

Por otra parte estos entornos también desafían las prácticas educativas existentes y consolidadas, que se han utilizado durante décadas, si no siglos, e impulsan a los docentes y expertos educativos fuera de su zona de confort haciéndoles conscientes de las limitaciones de las prácticas de enseñanza y evaluación vigentes y la evidencia de que pueden mejorarse a través de estos nuevas posibilidades.

La filosofía, las convicciones, los patrones consolidados de análisis y de comportamiento que ilustran las políticas universitarias existentes también se convierten en una barrera frente a la mejora de la educación universitaria y de las expectativas personales que estos entornos permiten. $Y$ eso es así en función de que estas prácticas están orientadas y determinadas por viejos y caducos paradigmas educativos. Hacer frente a estos desafíos requerirá esfuerzos concertados de las diversas partes interesadas en los diferentes niveles: Docentes a nivel de base, responsables de la gestión universitaria, políticos y, en realidad, de todas las personas, de la industria, los servicios y de las profesiones.

Presentación del artículo: 15 de marzo de 2018 Fecha de aprobación: 28 de marzo de 2018 Fecha de publicación: 31 de marzo de 2018

\section{Financing}

This research has not received any specific subsidies from funding agencies in the public, commercial or non-profit sectors.

\section{Financiación}

Esta investigación no ha recibido ninguna subvención específica de los organismos de financiación en los sectores públicos, comerciales o sin fines de lucro.

Zapata-Ros, M. (2018). La universidad inteligente. RED. Revista de Educación a

Distancia, 57(10). Consultado el (dd/mm/aaaa) en

http://www.um.es/ead/red/57/zapata2.pdf 


\section{Referencias}

Bloom, B. (1984). The 2 Sigma Problem: The Search for Methods of Group Instruction as effective as One-to-One Tutoring, Educational Researcher, 13:6 (4-16).

Brandman, R. (2013) 5 Tips: Learn more effectively in class with Mastery Learning. Course Operations MOOC Pedagogy Specialist, Accesible el 09/06/13 en http://blog.coursera.org/post/50352075945/5-tips-learn-more-effectively-in-classwith-mastery

Brown, J. S., Collins, A., \& Duguid, P. (1989). Situated cognition and the culture of learning. Educational researcher, 18(1), 32-42.

Bomsdorf, B. (2005). Adaptation of learning spaces: Supporting ubiquitous learning in higher distance education. In Dagstuhl Seminar Proceedings. Schloss Dagstuhl-Leibniz-Zentrum fr Informatik.

Bothwell, E. (March 6, 2018). Coursera to offer degrees from UK universities. Times Higher Education https://www.timeshighereducation.com/news/coursera-offer-degrees-ukuniversities.

Brusilovsky, P. (1996). Methods and techniques of adaptive hypermedia. User modeling and user-adapted interaction, 6(2-3), 87-129.

Chen, N. S., Cheng, I. L., \& Chew, S. W. (2016). Evolution is not enough: Revolutionizing current learning environments to smart learning environments. International Journal of Artificial Intelligence in Education, 26(2), 561-581. https://link.springer.com/article/10.1007\%2Fs40593-016-0108-x. En abierto https://www.researchgate.net/publication/295186361_Evolution_Is_not_enough_Revolu tionizing_Current_Learning_Environments_to_Smart_Learning_Environments

Chen, Y. L., Cheng, L. C., \& Chuang, C. N. (2008). A group recommendation system with consideration of interactions among group members. Expert systems with applications, 34(3), 2082-2090.

Chu, H. C., Hwang, G. J., \& Tsai, C. C. (2010). A knowledge engineering approach to developing mindtools for context-aware ubiquitous learning. Computers \& Education, 54(1), 289-297.

Chiou, C. K., Tseng, J. C., Hwang, G. J., \& Heller, S. (2010). An adaptive navigation support system for conducting context-aware ubiquitous learning in museums. Computers \& Education, 55(2), 834-845.

Clancey, W. J. (1984). Methodology for building an intelligent tutoring system. Methods and tactics in cognitive science, 51-84.

Clark, D. (February 19, 2016) 10 powerful results from Adaptive (AI) learning trial at ASU. Plan B. http://donaldclarkplanb.blogspot.com.es/2016/02/10-powerful-results-from-adaptiveai.html

Clark, D. (JANUARY 13, 2016). 5 level taxonomy of Al in learning (with real examples). Plan B. http://donaldclarkplanb.blogspot.com.es/search?q=ai+taxonomy

Clark, R. C. (2003). Building Expertise: Cognitive Methods for Training and Performance Improvement. Washington D.C., International Society for Performance Improvement.

Clark, R. C. and R. E. Mayer (2003). E-Learning and the Science of Instruction. San Francisco, Jossey-Bass Pfeiffer. 
Crosslin, M (2014 May). Designing a Dual Layer cMOOC/xMOOC. http://www.edugeekjournal.com/2014/05/04/designing-a-dual-layer-cmoocxmooc/

Dabbagh, N., \& Kitsantas, A. (2012). Personal Learning environments, social media, and selfregulated learning: A natural formula for connecting formal and informal learning. The Internet and Higher Education, 15(1), 3-8.

Delgado, C. et al (2017) Informe Tendencias TIC. Publicaciones de la CRUE. http://tic.crue.org/wp-content/uploads/2017/11/9.50-Sesion-Tecnica-CRUE-TIC-GTDIRTendencias-UCM-2017v1.pdf

Driscoll, M. (1994). Gagne's theory of instruction. Psychology of Learning for Instruction. Boston, MA, Allyn and Bacon, 329-358.

http://ocw.metu.edu.tr/pluginfile.php/9013/mod_resource/content/1/driscollch10\%20(1).pdf

Driscoll, M. P. (2013). Psychology of Learning for Instruction: Pearson New International Edition. Pearson Higher Ed.

FEFC (1997). How to Apply for Funding. Bristol: Further Education Funding Council.

Fink, L.D. (2003), A Self-Directed Guide to Designing Courses for Significant Learning.http://www.deefinkandassociates.com/GuidetoCourseDesignAug05.pdf

Gagné, R. M. (1965). The conditions of learning and theory of instruction (1st ed.). New York, NY: Holt, Rinehart \& Winston.

Gagné, R. M., \& Briggs, L. J. (1974). The principles of instructional design ( 1st ed.). New York, NY: Holt.

Gagné, R. M. (1985). The conditions of learning and theory of instruction (4th ed.). New York, NY: Holt, Rinehart \& Winston.

Goel, A. K., \& Polepeddi, L. (2016). Jill Watson: A Virtual Teaching Assistant for Online Education. Georgia Institute of Technology. https://smartech.gatech.edu/bitstream/handle/1853/59104/goelpolepeddiharvardvolume-v7.1. pdf?sequence=1\&isAllowed=y

Gonzalez, A. J., \& Ingraham, L. R. (1994). Automated exercise progression in simulation-based training. IEEE transactions on systems, man, and cybernetics, 24(6), 863-874.

Graf, S., \& Ives, C. (2010, July). A flexible mechanism for providing adaptivity based on learning styles in learning management systems. In Advanced Learning Technologies (ICALT), 2010 IEEE 10th International Conference on (pp. 30-34). IEEE.

Hsieh, S. W., Jang, Y. R., Hwang, G. J., \& Chen, N. S. (2011). Effects of teaching and learning styles on students' reflection levels for ubiquitous learning. Computers \& Education, 57(1), 1194-1201.

Hung, P. H., Hwang, G. J., Lin, Y. F., Wu, T. H., \& Su, I. H. (2013). Seamless Connection between Learning and Assessment-Applying Progressive Learning Tasks in Mobile Ecology Inquiry. Educational Technology \& Society, 16(1), 194-205.

Hung, I. C., Yang, X. J., Fang, W. C., Hwang, G. J., \& Chen, N. S. (2014). A context-aware video prompt approach to improving students' in-field reflection levels. Computers \& Education, 70, 80-91.

Hwang, G. J. (1998). A tutoring strategy supporting system for distance learning on computer networks. IEEE TRANSACTIONS ON EDUCATION E, 41, 343-343. 
Hwang, G. J., \& Chang, H. F. (2011). A formative assessment-based mobile learning approach to improving the learning attitudes and achievements of students. Computers \& Education, 56(4), 1023-1031.

Hwang, G. J., Tsai, C. C., Chu, H. C., Kinshuk, K., \& Chen, C. Y. (2012). A context-aware ubiquitous learning approach to conducting scientific inquiry activities in a science park. Australasian Journal of Educational Technology, 28(5).

Hwang, G. J. (2014). Definition, framework and research issues of smart learning environments-a context-aware ubiquitous learning perspective. Smart Learning Environments, 1(1), 4. https://slejournal.springeropen.com/articles/10.1186/s40561-0140004-5

Hwang, G. J., Chin-Chung, T., \& Yang, S. J. (2008). Criteria, strategies and research issues of context-aware ubiquitous learning. Journal of Educational Technology \& Society, 11(2).

Hwang, G. J., Hung, P. H., Chen, N. S., \& Liu, G. Z. (2014). Mindtool-assisted in-field learning (MAIL): An advanced ubiquitous learning project in Taiwan. Journal of Educational Technology \& Society, 17(2).

Joiner, R., Nethercott, J., Hull, R., \& Reid, J. (2006). Designing Educational Experiences Using Ubiquitous Technology. Computers in Human Behavior, 22 (1), 67-76.

Jonassen, D. H., Carr, C., \& Yueh, H. P. (1998). Computers as mindtools for engaging learners in critical thinking. TechTrends, 43(2), 24-32.

Karampiperis, P., \& Sampson, D. (2005). Adaptive learning resources sequencing in educational hypermedia systems. Journal of Educational Technology \& Society, 8(4).

Kinshuk, T. L., \& Patel, A. (2003). User exploration based adaptation in adaptive learning systems. International Journal of Information Systems in Education, 1(1), 22-31.

Kinshuk, K., Hwang, G. J., Tsai, C. C., Chu, H. C. \& Chen, C. Y. (2012). A context-aware ubiquitous learning approach to conducting scientific inquiry activities in a science park. Australasian Journal of Educational Technology, 28(5).

Kinshuk (2014). Roadmap for adaptive and personalized learning in Ubiquitous environments. In Ubiquitous Learning Environments and Technologies (pp. 1-13). Heidelberg: Springer.

Kinshuk (2015). Smart learning/ecology of education/.... Personal blog. Retrieved from http://www.kinshuk.info/2015/10/smart-learning-ecology-of-education/

Lave, J. (1991). Situating learning in communities of practice. Perspectives on socially shared cognition, 2, 63-82.

Linden, A., \& Fenn, J. (2003). Understanding Gartner's hype cycles. Strategic Analysis Report No R-20-1971. Gartner, Inc.

Liu, G. Z., \& Hwang, G. J. (2010). A key step to understanding paradigm shifts in e-learning: towards context-aware ubiquitous learning. British Journal of Educational Technology, 41(2).

Mampadi, F., Chen, S. Y., Ghinea, G., \& Chen, M. P. (2011). Design of adaptive hypermedia learning systems: A cognitive style approach. Computers \& Education, 56(4), 1003-1011.

Martens, A., \& Uhrmacher, A. M. (2002, June). Adaptive tutoring processes and mental plans. In International Conference on Intelligent Tutoring Systems (pp. 71-80). Springer, Berlin, Heidelberg.

Martens, A. (2006). Modeling of adaptive tutoring processes. Web-based intelligent e-learning systems: Technologies and applications, 193-215. 
Merrill, M. D. (1991). Constructivism and instructional design. Educational technology, 31(5), 45-53.

Merrill, M. D. (2002). First principles of instruction. Educational Technology Research and Development, 50(3), 43-59. (Click for copy from Dr. Merrill's personal website). Instructional-Design Theories and Models, Volume III: Building a Common Knowledge Base (link to book site)

Merrill, M. D. (2009). First Principles of Instruction. In C. M. Reigeluth \& A. Carr (Eds.), Instructional Design Theories and Models: Building a Common Knowledge Base (Vol. III). New York: Routledge Publishers. (Click for copy)

Minami, M., Morikawa, H., \& Aoyama, T. (2004, January). The design of naming-based service composition system for ubiquitous computing applications. In Applications and the Internet Workshops, 2004. SAINT 2004 Workshops. 2004 International Symposium on (pp. 304-312). IEEE.

Ogata, H., \& Yano, Y. (2004). Context-aware support for computer-supported ubiquitous learning. In Wireless and Mobile Technologies in Education, 2004. Proceedings. The 2nd IEEE International Workshop on (pp. 27-34). IEEE.

Papanikolaou, K. A., Grigoriadou, M., Magoulas, G. D., \& Kornilakis, H. (2002). Towards new forms of knowledge communication: the adaptive dimension of a web-based learning environment. Computers \& Education, 39(4), 333-360.

Parr, C. (February 28, 2013). Universities mine institutional data in search of gold Analysis of information on staff and students can help to improve recruitment and retention. Times Higher Education. https://www.timeshighereducation.com/news/universities-mineinstitutional-data-in-search-of-gold/2002053.article.

Pazzani, M. J., \& Billsus, D. (2007). Content-based recommendation systems. In The adaptive web (pp. 325-341). Springer, Berlin, Heidelberg.

Reigeluth, C. M., Ed. (1999). Instructional-Design Theories and Models: A New Paradigm of Instructional Theory. Mahwah, NJ, Lawrence Erlbaum Associates Publishers.

Reigeluth, C. (2016). Teoría instruccional y tecnología para el nuevo paradigma de la educación. RED. Revista de Educación a Distancia. Número 50. 30 de septiembre de 2016. Consultado el (dd/mm/aaa) en http://www.um.es/ead/red/50

Reigeluth, C. M., \& Carr-Chellman, A. A. (2009a). Situational principles of instruction. In C. M. Reigeluth \& A. A. Carr-Chellman (Eds.), Instructional-design theories and models: Building a common knowledge base (Vol. III, pp. 57-68). New York: Routledge.

Rogers, Y., Price, S., Randell, C., Fraser, D. S., Weal, M., \& Fitzpatrick, G. (2005). Ubi-learning integrates indoor and outdoor experiences. Communications of the ACM, 48(1), 55-59.

Sharples, M., Sánchez, I. A., Milrad, M., \& Vavoula, G. (2009). Mobile Learning: Small devices, Big issues. In and SBS Balacheff, S. Ludvigsen, T. de Long, A. Lazonder (Ed.), Technology enhanced learning: Principles and products (pp. 233-49).

Siemens, G. (2014, May). Multiple pathways: Blending xMOOCs \& cMOOCs. http://www.elearnspace.org/blog/2014/05/06/multiple-pathways-blendingxmoocscmoocs/

Specht, M., Weber, G., Heitmeyer, S., \& Schöch, V. (1997, June). AST: adaptive WWWcourseware for statistics. In Proceedings of Workshop" Adaptive Systems and User Modeling on the World Wide Web" at 6th International Conference on User Modeling, UM97, Chia Laguna, Sardinia, Italy (pp. 91-95). 
Tsai, P. S., Tsai, C. C., \& Hwang, G. J. (2012). Developing a survey for assessing preferences in constructivist context-aware ubiquitous learning environments. Journal of computer assisted learning, 28(3), 250-264.

Tseng, J. C., Chu, H. C., Hwang, G. J., \& Tsai, C. C. (2008a). Development of an adaptive learning system with two sources of personalization information. Computers \& Education, 51(2), 776-786.

Tseng, S. S., Jun-Ming, S., Hwang, G. J., Hwang, G. H., Chin-Chung, T., \& Tsai, C. J. (2008b). An object-oriented course framework for developing adaptive learning systems. Journal of Educational Technology \& Society, 11(2).

Van Seters, J. R., Ossevoort, M. A., Tramper, J., \& Goedhart, M. J. (2012). The influence of student characteristics on the use of adaptive e-learning material. Computers \& Education, 58(3), 942-952.

Wu, H. K., Lee, S. W. Y., Chang, H. Y., \& Liang, J. C. (2013a). Current status, opportunities and challenges of augmented reality in education. Computers \& education, 62, 41-49.

Wu, P. H., Hwang, G. J., \& Tsai, W. H. (2013b). An expert system-based context-aware ubiquitous learning approach for conducting science learning activities. Journal of Educational Technology \& Society, 16(4).

Yang, C. C., Hwang, G. J., Hung, C. M., \& Tseng, S. S. (2013a). An evaluation of the learning effectiveness of concept map-based science book reading via mobile devices. Journal of Educational Technology \& Society, 16(3).

Yang, T. C., Hwang, G. J., \& Yang, S. J. H. (2013b). Development of an adaptive learning system with multiple perspectives based on students' learning styles and cognitive styles. Journal of Educational Technology \& Society, 16(4), 185.

ZAPATA-ROS, M. (1990) Técnicas de programación declarativa en el aula. Seco Olea Ediciones, SL Madrid.

Zapata-Ros, M. (2001). Formación abierta ya distancia a través de redes digitales: Modelos de redes de aprendizaje. RED: Revista de Educación a Distancia, (1).

Zapata-Ros, M. (2012). Calidad en entornos ubicuos de aprendizaje. RED: Revista de Educación a Distancia, (31), 1-12.

Zapata-Ros, M. (2015). Teorías y modelos sobre el aprendizaje en entornos conectados y ubicuos. Bases para un nuevo modelo teórico a partir de una visión crítica del "conectivismo". Teoría de la Educación. Educación y Cultura en la Sociedad de la Información, 16(1).

Zapata-Ros, M. (2015b). El diseño instruccional de los MOOC y el de los nuevos cursos abiertos personalizados. Revista de Educación a Distancia, (45).

Zapata-Ros, M. (2017, December). Latinoamérica y la educación superior en la encrucijada de la sociedad del conocimiento. Desafíos y disrupciones. Virtualidad, Educación y Ciencia, 8(15), 193-199.

Zapata-Ros, M. (2017). Latinoamérica y la educación superior en la encrucijada de la Sociedad del Conocimiento. Desafíos y disrupciones. RED de Hypotheses. Retrieved from https://red.hypotheses.org/1011 